\title{
Some Networks That Can Learn, Remember, and Reproduce Any Number of Complicated Space-Time Patterns, I
}

\section{STEPHEN GROSSBERG*}

\author{
Communicated by Gian-Carlo Rota
}

Page

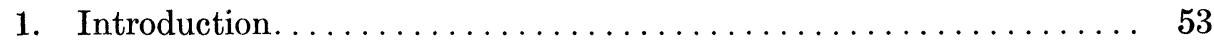

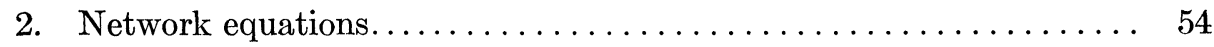

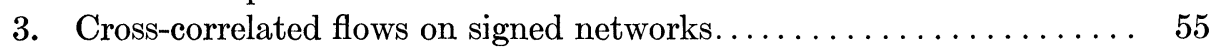

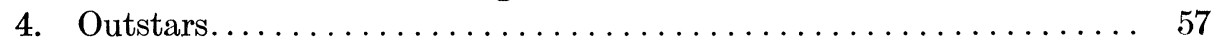

5. Learning a single spatial pattern of arbitrary complexity........ 59

6. Respondant conditioning........................ 61

7. Complete graph with loops: Pattern completion and the rigidityplasticity continuum...................... 62

8. Approximating any continuous space-time pattern by a series of

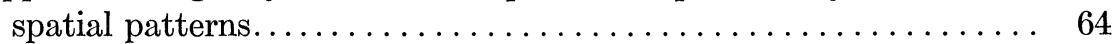

9. A better approximation: Signal thresholds elimate source noise. . . . . 67

10. Simultaneous storage of patterns and multimodal learning. . . . . . 80

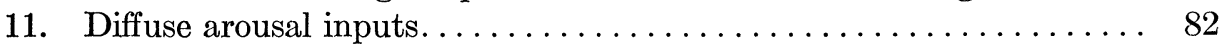

12. Feedback inhibition from control vertices................ 84

13. A hierarchy of facilitatory and incompatible behavioral acts...... 85

14. Novelty and habituation....................... 88

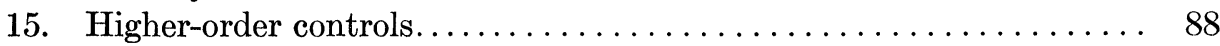

16. "On"-"Off" performance of complicated reflexes and "paying atten-

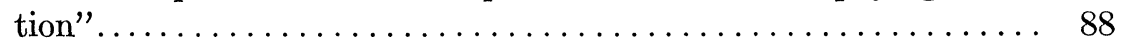

17. Alternative systems....................... 90

1. Introduction. This paper describes some networks $\mathfrak{T C}$ that can learn, simultaneously remember, and individually reproduce on demand any number

* The preparation of this work was supported in part by the National Science Foundation (GP 9003) and the Office of Naval Research (N00014-67-A-0204-0016). 
of spatiotemporal patterns (e.g., "motor sequences") of essentially arbitrary complexity. Because these networks are embedding fields, their behavior can be psychologically, neurophysiologically, and anatomically interpreted ([1], [2], [3], [4]). The network properties include the following.

(a) "Practice makes perfect".

(b) Memory of each pattern is essentially perfect if no competing experimental practice is imposed.

(c) New patterns can be learned without at all destroying the memory of old patterns.

(d) All errors can be corrected.

(e) No "subject-induced" overt or covert practice is needed to ensure perfect memory.

(f) Given a moderate amount of practice, memory spontaneously improves (i.e., "reminiscence" occurs).

(g) Memory is not destroyed by recall trials.

(h) Learning occurs by a mixture of respondant and operant conditioning, the operant effects including nonspecific arousal inputs in response to "novel" stimuli, and induced blocking of incoming inputs by inhibitory signals, leading to "habituation" of repeated inputs. Both respondant and operant factors are unified into a single comprehensive learning mechanism.

(i) Only one "control neuron" is needed to activate reproduction of an entire space-time pattern.

(j) The time needed to begin recall of a pattern can be made as small as we please, and is independent of pattern complexity.

(k) The network is insensitive to wild "behaviorally irrelevant" oscillations of inputs.

(l) The network dynamics, though nonlinear, can be analysed globally.

Networks that perform any number of complicated "reflex acts" (e.g., "walking", "clasping", "sniffing") will also be constructed, as a special case of the learning networks. These "reflex" networks also satisfy (i)-(l) above.

2. Network equations. The networks we will use were derived in [2] and [3], and are reviewed in [1]. They are a special case of the following equations.

$$
\begin{gathered}
\dot{x}_{i}(t)=-\alpha_{i} x_{i}+\sum_{m=1}^{n} \beta_{m}\left[x_{m}\left(t-\tau_{m i}\right)-\Gamma_{m i}\right]^{+} y_{m i}(t) \\
-\sum_{m=1}^{n}\left[x_{m}\left(t-\tau_{m i}\right)-\Gamma_{m i}\right]^{+} q_{m i}+I_{i}(t), \\
y_{j k}(t)=p_{i k} z_{j k}(t)\left[\sum_{m=1}^{n} p_{j m} z_{j m}(t)\right]^{-1}, \text { and } \\
\left({ }^{*}\right) \\
\text { (3) } \quad \dot{z}_{j k}(t)= \begin{cases}-u_{i k} z_{j k}(t)+v_{j k}\left[x_{j}\left(t-\tau_{i k}\right)-\Gamma_{i k}\right]^{+} x_{k}(t), & \text { if } p_{i k}>0 \\
0, & \text { if } p_{i k}=0,\end{cases}
\end{gathered}
$$


for $i, j, k=1,2, \cdots, n$ where

$$
[\eta]^{+}=\max (\eta, 0)
$$

for any real number $\eta$. The parameters, initial data, and inputs of $\left(^{*}\right)$ satisfy the following constraints.

(I) Parameters:

(1) All constant parameters are nonnegative; e.g., $\alpha_{i}, \beta_{m}, \Gamma_{m i}, p_{i k}$.

(2) The $n \times n$ matrix $P=\left\|p_{i k}\right\|$ is semi-stochastic; i.e., $p_{i k} \geqq 0$ and $\sum_{m=1}^{n} p_{i m}=0$ or 1 .

(3) $v_{j k}$ is positive only if $p_{j k}$ is positive.

(4) All time lags $\tau_{j k}$ are positive.

(5) $p_{m i} q_{m i}=0$ for all $i, m=1,2, \cdots, n$.

(II) Initial Data. All initial data of $x_{i}(v)$ and $z_{j k}(v)$ for $v \leqq 0$ is nonnegative and continuous. Moreover we suppose for convenience that $z_{i k}(0)>0$ if and only if $p_{i k}>0$.

(III) Inputs. All inputs $I_{i}(t)$ are bounded, nonnegative, and continuous for $t \geqq 0$ and vanish for $t<0$.

When we say henceforth that parameters, initial data, or inputs are chosen "arbitrarily", we will always mean "arbitrarily subject to (I)-(III)".

3. Cross-correlated flows on signed networks. The equations (1)-(3) describe a cross-correlated flow on a signed network $\mathfrak{T}$. Since variants of this flow have been previously described in several places ([1]-[13]), the following summary will be brief.

Let a finite directed graph $G=(V, E)$ be given with vertices $V=\left\{v_{i}: i=\right.$ $1,2, \cdots, n\}$ and directed edges $E=\left\{e_{j k}: j, k=1,2, \cdots, n\right\} . e_{j k}$ is drawn as an arrow facing from the point $v_{j}$ whose arrowhead $N_{j k}$ touches the point $v_{k} \cdot x_{i}(t)$ describes a process at $v_{i}$, whereas $z_{j k}(t)$ and $y_{j k}(t)$ describe processes at $N_{j k}$. At every time $t-\tau_{m i}$, the value $x_{m}\left(t-\tau_{m i}\right)$ at $v_{m}$ creates an excitatory signal of size

$$
\beta_{m}\left[x_{m}\left(t-\tau_{m i}\right)-\Gamma_{m i}\right]^{+} p_{m i} .
$$

In particular, the signal (5) is positive only if the path weight $p_{m i}$ is positive and if $x_{m}\left(t-\tau_{m i}\right)$ is greater than the signal threshold $\Gamma_{m i}$. The signal (5) travels at a finite velocity along $e_{m i}$ and reaches the arrowhead $N_{m i}$ at time $t$. It thereupon interacts with the processes $z_{m i}$ and $y_{m i}$, and a signal of size

$$
\beta_{m}\left[x_{m}\left(t-\tau_{m i}\right)-\Gamma_{m i}\right]^{+} y_{m i}(t)
$$

is released from $N_{m i}$ and reaches $v_{i}$, where it perturbs $x_{i}$. All signals from the various $v_{m}$ combine additively at $v_{i}$, yielding the second term on the right hand side of (1). $x_{i}(t)$ also decays exponentially at a rate $\alpha_{i}$, and is perturbed by the input $I_{i}(t)$ which is under the control of an experimentalist or other external environmental factors. 
An inhibitory signal of size

$$
\left[x_{m}\left(t-\tau_{m i}\right)-\Gamma_{m i}\right]^{+} q_{m i}
$$

leaves $v_{m}$ at time $t-\tau_{m i}$. By (I5) of Section 2, either (5) or (7) is identically zero.

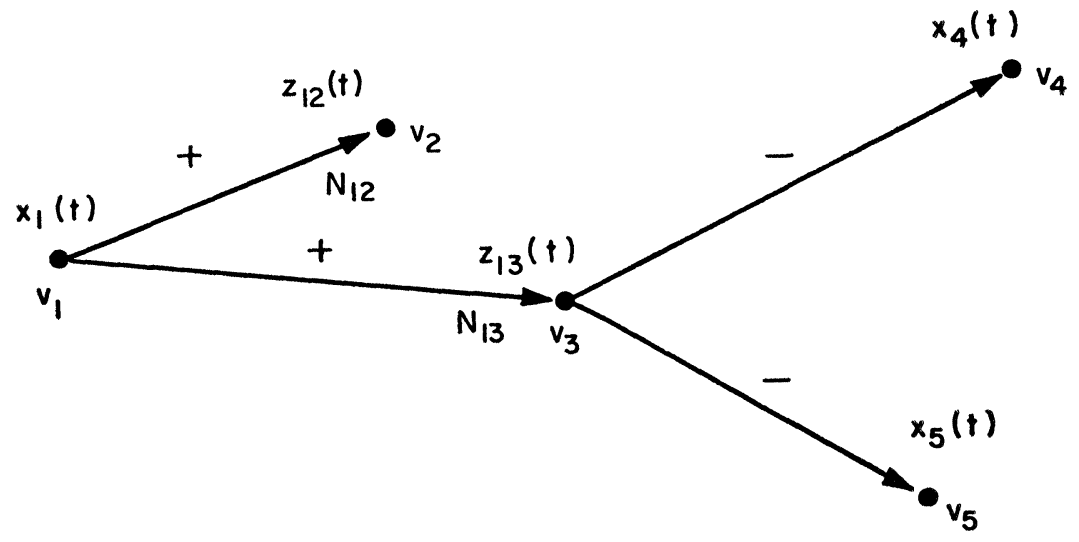

Figure 1.

$z_{m i}(t)$ sits in $N_{m i}$ and cross-correlates the signal (5) received by $N_{m i}$ at time $t$ with the contiguous value $x_{i}(t)$ of $v_{i}$; hence the condition (I3). $z_{m i}(t)$ also decays exponentially at the rate $u_{j k}$, which can be zero in special cases (see [5]).

$y_{m i}(t)$, rather than $z_{m i}(t)$, controls the excitatory signal that reaches $v_{i}$ from $v_{m} . y_{m i}$ is a ratio of cross-correlators, as in (2). This ratio can be interpreted as a kind of "competition between associations" or as "lateral inhibition coupled to cross-correlators", and is helpful in some form to make perfect learning possible ([1], [2], [3]). [13] describes systems where $z_{m i}$ replaces $y_{m i}$.

Each of the dynamical variables in $(*)$ has a mathematical, psychological, and neural name. Thus

$$
\begin{aligned}
x_{i} & =i^{\text {th }} \text { vertex function, or } \\
& =i^{\text {th }} \text { stimulus trace, or } \\
& =i^{\text {th }} \text { average membrane potential, }
\end{aligned}
$$

and

$$
\begin{aligned}
y_{j k} & =(j, k)^{\text {th }} \text { edge (or interaction) function, or } \\
& =\text { associational strength from } v_{i} \text { to } v_{k} \text {, or } \\
& =\text { average activity of transmitter control process in } N_{i k} .
\end{aligned}
$$

The network components also have qualitative anatomical labels. Thus

$$
\begin{aligned}
v_{i} & =i^{\text {th }} \text { cell body cluster, } \\
e_{i k} & =\text { cluster of axons from } v_{i} \text { to } v_{k}, \text { and } \\
N_{i k} & =\text { cluster of synaptic knobs at terminal ends of } e_{i k} \text { axons. }
\end{aligned}
$$


Each choice of the matrices $P=\left\|p_{i k}\right\|$ and $Q=\left\|q_{i k}\right\|$ defines a different "anatomy" for a network IT by picking out the directed paths $v_{i} \rightarrow v_{k}$ over which signals can be transmitted and the relative strengths of these signals. Varying $P$ and $Q$ can dramatically change the qualitative properties of learning, memory, and recall in a network ([5]-[13]). The task of this paper is, given (1)-(3), mainly to choose an "anatomy" $(P, Q)$ that can accomplish the paper's stated task. This will not be the only anatomy that can do the job, but it will certainly be a very simple one, and its deficiencies, where they exist, must be remedied by passing to more elaborate anatomies; see [12] for an example of a more realistic anatomy.

4. Outstars. An especially simple example of $(*)$ is given by the equations

$$
\begin{aligned}
& \dot{x}_{1}(t)=-\alpha x_{1}(t)+I_{1}(t), \\
& \dot{x}_{i}(t)=-\alpha x_{i}(t)+\beta x_{1}(t-\tau) y_{1 i}(t)+I_{i}(t),
\end{aligned}
$$

and

$$
y_{1 i}(t)=z_{1 i}(t)\left[\sum_{m=2}^{n} z_{1 m}(t)\right]^{-1}
$$

where $i=2,3, \cdots, n$. $\left({ }^{* *}\right)$ is characterized by the following parameter choices in $\left(^{*}\right)$.
a) $\alpha_{i}=\alpha$,
b) $\beta_{i}=\beta$,
c) $u_{j k}=u$,
d) $v_{j k}=\beta$,
e) $\Gamma_{i k}=q_{i k}=0$, and

$$
\text { f) } p_{i i}=\left\{\begin{array}{lll}
0, & \text { unless } & i=1 \\
0, & \text { if } & i=j=1 \\
\frac{1}{n-1}, & \text { if } & i=1 \neq j .
\end{array}\right.
$$

The probabilistic graph characterized by (12) is drawn in Figure 2.

Hence the network $\mathfrak{T}^{(1)}$ which obeys $\left.{ }^{(* *}\right)$ is called an outstar with source vertex $v_{1}$, sink vertices $v_{i}, i \neq 1$, and border $B_{n}=\left\{v_{i}: i=2, \cdots, n\right\}$. We will construct the network of this paper using several copies of suitably modified outstars.

The dynamics of $\mathfrak{T C}^{(1)}$ have been studied in [8] and are reviewed in [6]. These references describe the following theorem, which discusses an infinite sequence $G^{(1)}, G^{(2)}, \cdots, G^{(N)}, \cdots$ of outstars. Each outstar represents a "learning subject" who is matched in prior learning experience (i.e., initial data) with all other subjects. $G^{(N)}$ differs from $G^{(N-1)}$ only by receiving more practice than 


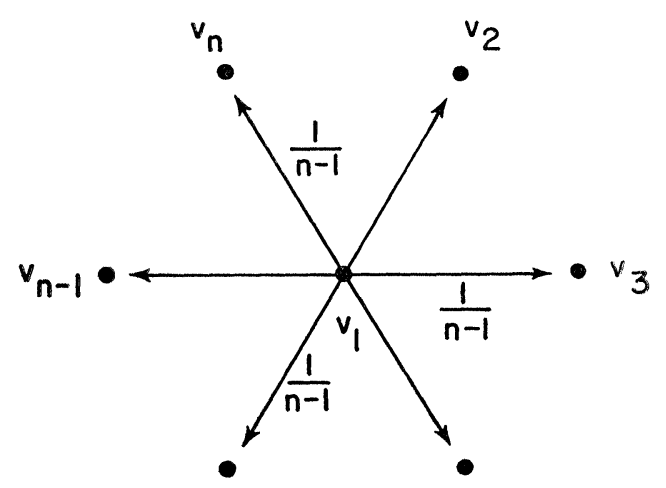

Frgure 2.

$G^{(N-1)}$, and by perhaps being subjected to a different sequence of recall trials. The theorem means heuristically that in an outstar,

1) "practice makes perfect",

2) an isolated outstar suffers no memory loss,

3) an isolated outstar remembers without overtly practicing,

4) after moderate amounts of practice, the memory of an outstar spontaneously improves,

5) the act of making a correct recall can be repeated as many times as one wishes without destroying the outstar's memory, and

6) all errors can be corrected, although the retraining time might be lengthened due to response interference.

The theorem discusses the probabilities $y_{1 i}(t)$ of $(10)$ and the correspondingly defined probabilities

$$
X_{i}(t)=\frac{x_{i}(t)}{\sum_{m=2}^{n} x_{m}(t)},
$$

$i=2, \cdots, n$. We denote the $y_{1 i}, X_{i}$, and $I_{i}$ functions of $G^{(N)}$ by $y_{1 i}^{(N)}, X_{i}^{(N)}$, and $I_{i}^{(N)}$ respectively.

Theorem 1. Let $G^{(1)}, \cdots, G^{(N)}, \cdots$, be any sequence of outstars with equal but otherwise arbitrary initial data. Let $I_{i}^{(N)}$ be any inputs of the form

$$
I_{1}^{(N)}(t)=I_{1}(t) \chi\left(t-U_{1}(N)\right)+h_{N}(t)
$$

and

$$
I_{i}^{(N)}(t)=\theta_{i} I(t) \chi(t-U(N))
$$

$i=2, \cdots, n$, where

a) $\left\{\theta_{i}: i=2, \cdots, n\right\}$ is a fixed but arbitrary probability distribution (i.e., $\theta_{i} \geqq 0$ and $\sum_{i=2}^{n} \theta_{i}=1$ ); 
b) $U_{1}(N)$ and $U(N)$ are any nonnegative and strictly increasing functions of the integer $N \geqq 1$;

c) the inputs $I_{i}(t)$ and $I(t)$ are constrained only by the existence of positive constants $c$ and $T_{o}$ such that

$$
\int_{0}^{t} e^{-\alpha(t-v)} I_{1}(v) d v \geqq c
$$

and

$$
\int_{0}^{t} e^{-\alpha(t-v)} I(v) d v \geqq c
$$

for $t \geqq T_{o}$;

d) the input $h_{N}(t)$ is constrained only by being zero until $t>U(N)$; and

$$
\chi(t)= \begin{cases}1, & \text { if } t<0 \\ 0, & \text { if } t \geqq 0 .\end{cases}
$$

Then

A) for every $N \geqq 1$, the limits $Q_{i}^{(N)}=\lim _{t \rightarrow \infty} X_{i}^{(N)}(t)$ and $P_{1 i}^{(N)}=\lim _{t \rightarrow \infty} y_{1 i}^{(N)}(t)$ exist and are equal,

B) for every $N \geqq 1$ and $t \geqq U(N), X_{i}^{(N)}(t)$ and $y_{1 i}^{(N)}(t)$ are monotonic and are contained in the interval $\left[m_{i}^{(N)}, M_{i}^{(N)}\right]$, where

$$
\begin{aligned}
& m_{i}^{(N)}=\min \left(X_{i}^{(N)}(U(N)), y_{1 i}^{(N)}(U(N))\right), \\
& M_{i}^{(N)}=\max \left(X_{i}^{(N)}(U(N)), y_{1 i}^{(N)}(U(N))\right),
\end{aligned}
$$

and

$$
\lim _{N \rightarrow \infty} m_{i}^{(N)}=\lim _{N \rightarrow \infty} M_{i}^{(N)}=\theta_{i} .
$$

In particular, by (A) and (B),

$$
\lim _{N \rightarrow \infty} \lim _{t \rightarrow \infty} X_{i}^{(N)}(t)=\lim _{N \rightarrow \infty} \lim _{t \rightarrow \infty} y_{1 i}^{(N)}(t)=\theta_{i} ;
$$

$i=2, \cdots, n$.

C) for every $N \geqq 1$ and $i \neq 1$, the functions

$$
\dot{y}_{1 i}^{(N)}, f_{i}^{(N)}=y_{1 i}^{(N)}-X_{i}^{(N)} \text {, and } g_{i}^{(N)}=X_{i}^{(N)}-\theta_{i},
$$

change sign at most once and not at all if $f_{i}^{(N)}(0) g_{i}^{(N)}(0) \geqq 0$. Moreover $f_{i}^{(N)}(0) g_{i}^{(N)}(0)$ $>0$ implies that $f_{i}^{(N)}(t) g_{i}^{(N)}(t)>0$ and that $y_{1 i}^{(N)}(t)$ is monotonic for all $t \geqq 0$.

Remark. In [8], each $G^{(N)}$ is denoted by $G^{(N, f)}$ and Theorem 1 is Theorem 5(f).

5. Learning a single spatial pattern of arbitrary complexity. Theorem 1 allows us to use an outstar to learn a spatial pattern of arbitrary complexity. 
Suppose for example that we wish $\mathfrak{T}^{(1)}$ to learn the spatial pattern "A". First. we must specify the level of spatial descrimination that is desired. If, for example, $A$ is to be presented within a square region $R$, then we can arrange the $n-1$ border vertices $B_{n}$ of $\mathfrak{T}^{(1)}$ in a rectangular grid spread over $R$. The larger $n$ is taken, the greater will be $\mathfrak{T}^{(1)}$ 's ability to discriminate fine spatial details in the pattern. Since Theorem 1 holds for any value of $n=2,3, \cdots$, any prescribed level of spatial discrimination can be guaranteed. Figure 3 depicts the case $n=10$.

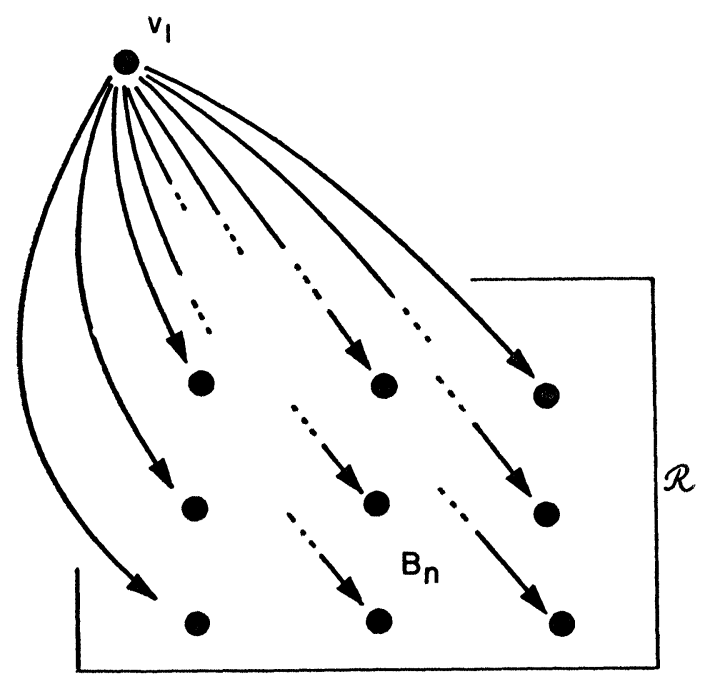

FigURe 3.

The "A-ness" of the pattern "A" does not depend on the absolute blackness of its lines, but only on their relative blackness as compared to the surround. A spatial pattern (in "black", "white", and "shades of gray") is therefore defined as an input function

$$
I_{i}(t)=\theta_{i} J(t), \quad i=2, \cdots, n,
$$

delivered to $B_{n}$, where the nonnegative number $\theta_{i}$ specifies the relative blackness of the portion of the pattern that is seen by $v_{i}$. The $\theta_{i}$ 's can clearly be normalized to form a probability distribution without loss of generality, and then $J(t)$ specifies the total intensity of the pattern at time $t$. The pattern " $\mathrm{A}$ " is the same whether or not we view it in steady light or in flickering light, within substantial physiological limits. $J(t)$ can thus oscillate quite wildly without changing the pattern described by the $\theta_{i}$ 's. Henceforth we therefore speak of the spatial pattern $\theta^{(n)}=\left\{\theta_{i}: i=2, \cdots, n\right\}$.

The inputs $I_{i}^{(N)}(t), i \neq 1$, in (15) are a spatial pattern $\theta^{(n)}$ with total intensity function

$$
J(t)=I(t) \chi(t-U(N)) .
$$


In other words, presentation of the pattern ceases at time $t=U(N)$. Theorem 1 guarantees that if the spatial pattern $\theta^{(n)}$ is presented sufficiently often (i.e., $N \rightarrow \infty)$, and if the source vertex $v_{1}$ is also perturbed sufficiently often, then a later perturbation of $v_{1}$ alone will recreate the spatial pattern $\theta^{(n)}$ on the grid.

6. Respondant conditioning. The above learning paradigm can readily be interpreted as a form of respondant conditioning. Consider Figure 4.

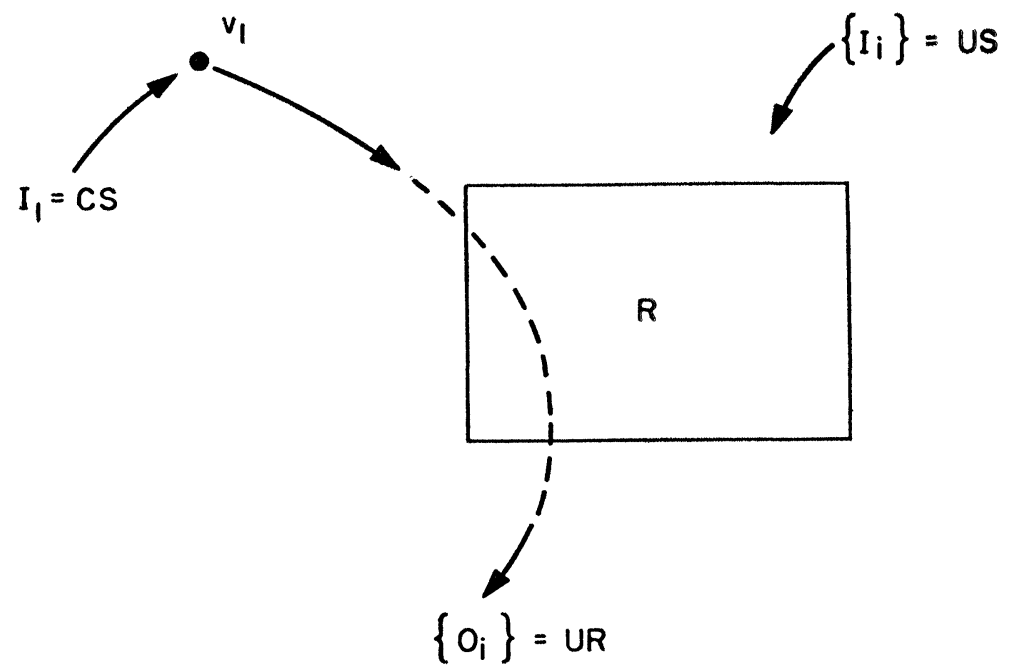

Figure 4.

Figure 4 emphasizes that inputs $I_{i}(t)$ reaching border vertices $v_{i}$ give rise to outputs $O_{i}(t)$ that are proportional to $x_{i}(t), i=2, \cdots, n$. Suppose moreover that all inputs $I_{i}(t)$ have equalled zero for a long time prior to $t=t_{o}$. Then by (8) and (9) we readily find that

$$
x_{i}\left(t_{o}\right) \cong 0, \quad i=1,2, \cdots, n
$$

(See [8], p. 673). Now let the spatial pattern $\theta^{(n)}$ perturb $B_{n}$ at times $t \geqq t_{o}$. Then by (8) and (9),

$$
x_{1}(t) \cong 0, \quad t \geqq t_{o}
$$

and thus

$$
\begin{aligned}
\dot{x}_{i}(t) & \cong-\alpha x_{i}(t)+I_{i}(t) \\
& =-\alpha x_{i}(t)+\theta_{i} J(t),
\end{aligned}
$$

$i=2, \cdots, n$, where $J(t)$ becomes positive after $t=t_{o}$. By (20) and (21),

$$
x_{i}(t) \cong \theta_{i} e^{-\alpha t} \int_{t_{0}}^{t} e^{\alpha v} J(v) d v,
$$


$i=2, \cdots, n,([8]$, pp. 675-677), and thus

$$
\frac{x_{i}(t)}{x_{i}(t)} \cong \frac{\theta_{i}}{\theta_{i}},
$$

for $i, j=2, \cdots, n$, and $t>t_{o}$. In other words, the spatial pattern input to $B_{n}$ creates the same spatial pattern output from $B_{n}$. We can therefore call the input pattern $I_{i}(t)$ an unconditioned stimulus (US) to $B_{n}$, and the output pattern $O_{i}(t)$ an unconditioned response (UR) from $B_{n}$ [14]. Learning in an outstar has the effect of producing the unconditioned response from $B_{n}$, given an input to $v_{1}$ alone, by previously pairing inputs to $v_{1}$ with unconditioned stimuli to $B_{n}$. If we call the input to $v_{1}$ a conditioned stimulus (CS), this learning procedure falls easily within the paradigm of respondant conditioning [14].

7. Complete graph with loops: pattern completion and the rigidity-plasticity continuum. [7] and [9] describe a different network that can also learn a spatial pattern of arbitrary complexity, namely a complete graph with loops, in which every $p_{i i}=1 / n$, all parameters are independent of their indices, and all thresholds and inhibitory signals equal zero. Learning in the complete graph with loops has some interesting properties that are not found in an outstar, such as the following ones.

a) Pattern completion. After the pattern is learned, even a single "speck of light" shined anywhere on the grid will suffice to reproduce the entire spatial pattern on the grid. The complete graph with loops is, in fact, the smallest embedding field that can do this.

b) Path rigidity vs. plasticity. Qualitative properties of memory in a complete graph with loops depend on whether or not the number $\sigma(\tau)$ is positive, where

$$
\sigma(\tau)=u+2 s(\tau)
$$

and $s(\tau)$ is the supremum of the real parts of the roots $s$ of the equation

$$
s+\alpha-\beta e^{-\tau s}=0 .
$$

If $\sigma(\tau)>0$, then

$$
\lim _{t \rightarrow \infty} y_{i i}(t)=\lim _{t \rightarrow \infty} \tilde{X}_{i}(t), \text { for all } i \text { and } j,
$$

where

$$
\widetilde{X}_{i}(t)=\frac{x_{i}(t)}{\sum_{m=1}^{n} x_{m}(t)}
$$

In other words, the associations converge to a spatial pattern in the absence of practice, and in fact to the spatial pattern which is "closest" to the graph's values $y_{i i}$ and $\tilde{X}_{i}$ when practice ceases. If $\sigma(\tau)<0$, this is false. For example, if $\tau=0$, then 


$$
\left|y_{j k}(t)-y_{j k}(0)\right| \leqq 2 \log \left(1+\frac{k_{i}}{|\sigma(0)|}\right),
$$

where $k_{j}$ depends on initial data. Thus by choosing $\sigma(\tau)<0$ and $|\sigma(\tau)|$ sufficiently large, the associations $y_{i k}(t)$ can be made to remember anything arbitrarily well. In this sense, the network is "plastic" for $\sigma(\tau)>0$ and "rigid" for $\sigma(\tau)<0$.

Suppose also that $\alpha>\beta$. This condition means heuristically that large outputs from the graph occur only in response to inputs, for any time lag $\tau \geqq 0$. In this case - which is the only case of physical interest-[9] shows that $\sigma(\tau)$ is a monotone increasing function of $\tau \geqq 0$. Hence if a way existed to gradually decrease the time lag $\tau$ as a function of time, then the sign of $\sigma(\tau)$ could be made to shift from positive to negative values if $\sigma(0)<0$. Since

$$
\sigma(0)=u+2(\beta-\alpha)
$$

this can happen only if

$$
0 \leqq u<2(\alpha-\beta)
$$

Supposing that the length of the edges remains fixed through time, decreasing $\tau$ means increasing the signal velocity along the edges. Thus an increase in signal velocity can take the network from a "plastic" memory phase to a "rigid" memory phase capable of better preserving old learning. Since also the best learning speeds approximate $\tau$ [2], increasing signal velocity also lets the network learn best at faster presentation rates.

In vivo, a standard method of increasing axonal signal velocity is to myelinate the axon [15]. Hence an abstract "myelinization" process, if coupled to the dynamics of learning in individual "cells" of a complete graph with loops, can help to speed up learning and to better preserve learned patterns in the cellular ensemble. A similar phenomenon occurs in complete graphs without loops (i.e., $p_{i j}=1 /(n-1)$ if $i \neq j$, and $\left.p_{i i}=0\right)$, at least in the case $\tau=0$ and $n=3$ [10]. Here if $\sigma(0)>0$, the graph forgets everything it has learned in the absence of overt practice, whereas if $\sigma(0)<0$ the graph can be made to remember arbitrarily well by choosing $|\sigma(0)|$ sufficiently large. Suppose, for example, that the excitation parameter $\beta$ and the decay parameter $\alpha$ are equal, but are chosen with any positive value. Then by (27), any choice $u>0$ of the correlational decay parameter, no matter how small, guarantees that $\sigma(0)>0$ and hence that the graph will eventually forget everything that it has learned. Such a graph can remain an "unbiased" input filter for all time if inputs are presented at a sufficiently slow rate compared to the decay parameter $\sigma(0)>0$, but can remember arbitrarily well if $\sigma(0)<0$ and $|\sigma(0)|$ is taken arbitrarily large.

Neither pattern completion nor dependence on the sign of $\sigma(\tau)$ occur in an outstar, which exhibits no pattern completion because $p_{i j}=0$ whenever $v_{i}$ is in $B_{n}$, and whose memory has the same qualitative properties for all $\tau \geqq 0$. Nonetheless, an outstar has a vital property which complete graphs lack. We will find that the outstar associations $y_{1 i}(t)$ will not learn patterns in the border 
$B_{n}$ during times $t$ for which the source function $x_{1}(t-\tau)$ is zero. Hence an outstar can be made to "sample" the patterns playing on $B_{n}$ at prescribed times $t$; namely, those times for which $x_{1}(t-\tau)$ is large. This cannot be done by complete graphs because perturbation of any vertex of a complete graph can indirectly perturb all other vertices via signals along the edges, as in pattern completion.

8. Approximating any continuous space-time pattern by a series of spatial patterns. A space-time pattern delivered to a grid of vertices $v_{i}$ is created by an arbitrary array of input functions $I_{i}(t), i=2, \cdots, n$. If the pattern weights

$$
\theta_{i}(t)=\frac{I_{i}(t)}{\sum_{m=2}^{n} I_{m}(t)},
$$

$i=2, \cdots, n$, vary "sufficiently" slowly, however, then we can approximate the space-time pattern by a series of spatial patterns in the following way.

Suppose for example that each function $\theta_{i}(t)$ varies very slowly in intervals of length $\xi$, where if some $\theta_{i}(t)$ is a rapidly varying function, then $\xi$ must be chosen sufficiently small. We wish to construct a network that samples the space-time pattern briefly every $\xi$ time units, and thereby learns this pattern as the series of spatial patterns

$$
\theta^{(n)}(k \xi), \quad k=1,2 \cdots,
$$

where at every fixed time $t, \theta^{(n)}(t)$ is the spatial pattern

$$
\left\{\theta_{i}(t): i=2, \cdots, n\right\} .
$$

If we can construct a network that samples briefly every $\xi$ time units for any fixed positive value of $\xi$, then our approximation of (29) by (28) can be made arbitrarily good, by continuity of the inputs $I_{i}(t)$.

A plausible proposal for doing this is readily suggested. Suppose that (29) is presented over a time interval of length $T$, and that the sampling interval $\xi$ is prescribed. Let

$$
K(\xi, T)=\left[\frac{T}{\xi}\right]+1
$$

where $[w]$ is the greatest integer less than $w$ for every real number $w . K(\xi, T)$ is the number of spatial patterns by which the space-time pattern will be successively approximated. Let $K(\xi, T)$ outstars

$$
\mathfrak{M}_{1}^{(1)}, \mathfrak{T}_{2}^{(1)}, \cdots, \mathfrak{M}_{K(\xi, T)}^{(1)}
$$

be given, and denote the vertex $v_{i}$ and functions $X_{i}, y_{i j}$, and $I_{i}$ of $\mathfrak{T T}_{k}^{(1)}$ by $v_{k, i}$, $X_{k, i}, y_{k, i i}$, and $I_{k, i}$, respectively. Suppose that

$$
v_{k, i}=v_{m, i}
$$

for every $k, m=1,2, \cdots, K(\xi, T)$, and $i=2, \cdots, n$; that is, each $\mathfrak{T}_{k}^{(1)}$ has the 
same border. Let $v_{k, 1}$ be perturbed by an input pulse of duration less than $\xi$ at a time $\xi$ units after $v_{k-1,1}$ is perturbed. See Figure 5.

Figure 5 depicts the series of outstars $\mathfrak{T T}_{k}^{(1)}$ whose source vertices are excited successively every $\xi$ time units by "axon collaterals" of the edge leading from $v_{0}$. The space-time pattern is presented to the common border $B_{n}$ of these outstars by an independent source of inputs and the border gives rise to outputs $O_{i}(t)$ as before. If the $k^{\text {th }}$ outstar could learn the $k^{\text {th }}$ spatial approximation to the space-time pattern, then a later input to $v_{0}$ alone would recreate the entire space-time pattern at $B_{n}$ by successively activating each source $v_{k, 1}, k=$ $1,2, \cdots, K(\xi, T)$. A single control vertex $v_{0}$ could hereby activate an arbitrarily complicated space-time pattern!

The equations describing the network of Figure 5 are

$$
\begin{aligned}
\dot{x}_{0}(t) & =-\alpha_{0} x_{0}(t)+I_{0}(t), \\
\dot{x}_{k, 1}(t) & =-\alpha_{1} x_{k, 1}(t)+\beta_{0} x_{0}(t-k \xi), \\
\dot{x}_{i}(t) & =-\alpha x_{i}(t)+\beta \sum_{k=1}^{K(\xi, T)} x_{k, 1}(t-\tau) y_{k, 1 i}(t)+I_{i}(t),
\end{aligned}
$$

$(* * *)$

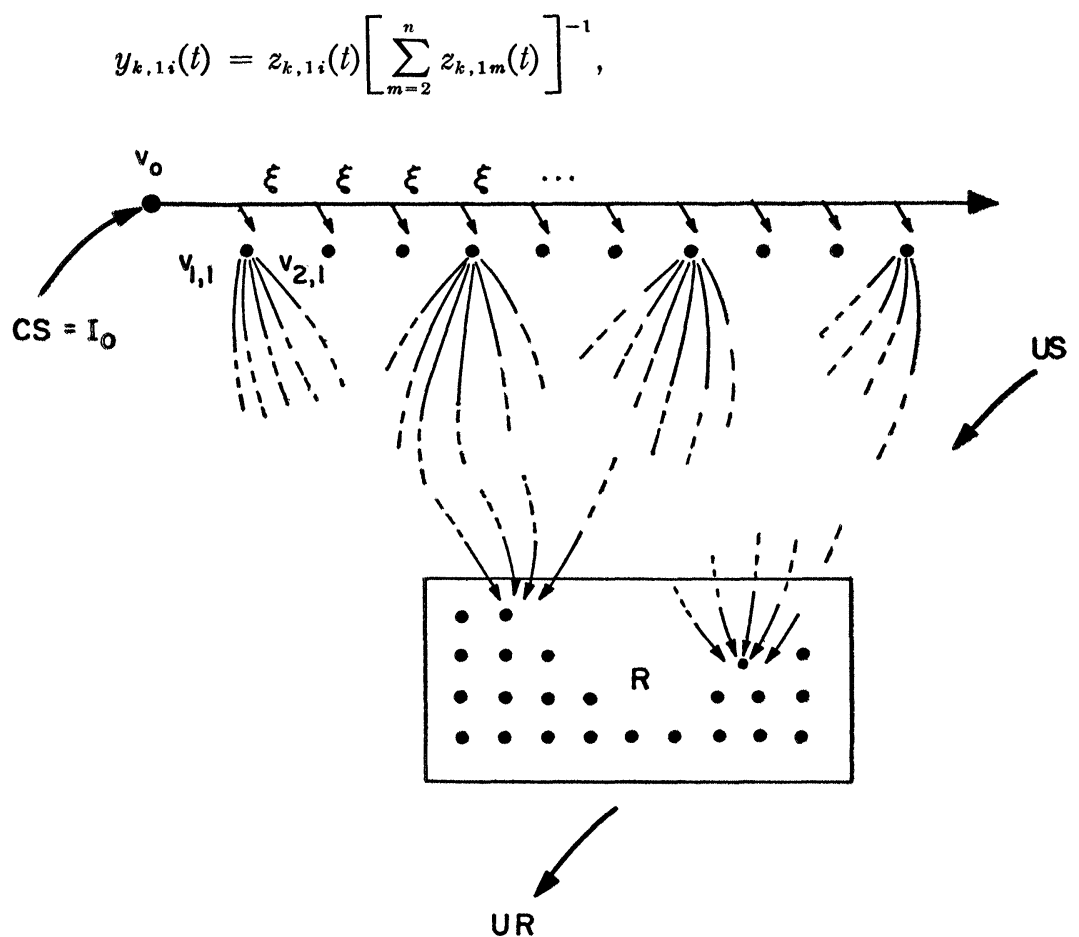

Figdre 5. 
and

$$
\dot{z}_{k, 1 i}(t)=-u z_{k, 1 i}(t)+\gamma x_{k, 1}(t-\tau) x_{i}(t),
$$

where $x_{i}(t)$ is the common value of all $x_{k, i}(t), k=1,2, \cdots, K(\xi, T)$, and $i=$ $2,3, \cdots, n .\left(^{* * *}\right)$ is called an outstar avalanche by analogy with Ramón y Cajal's description of parallel fiber conduction in the cerebellum as "avalanche" conduction ([16], p. 196).

Alternatively, $(* * *)$ can be replaced by the following system of equations:

$$
\begin{aligned}
\dot{x}_{0}(t) & =-\alpha_{0} x_{0}(t)+I_{0}(t) \\
\dot{x}_{k, 1}(t) & =-\alpha_{1} x_{k, 1}(t)+\beta_{0} x_{0}(t-k \xi), \\
\dot{x}_{k, i}(t) & =-\alpha x_{k, i}(t)+\beta x_{k, 1}(t-\tau) y_{k, 1 i}(t)+I_{i}(t), \\
y_{k, 1 i}(t) & =z_{k, 1 i}(t)\left[\sum_{m=2}^{n} z_{k, 1 m}(t)\right]^{-1}
\end{aligned}
$$

and

In this system, each $\mathfrak{T}_{k}^{(1)}$ has a separate grid, and the same input $I_{i}(t)$ is delivered to the $i^{\text {th }}$ border vertex $v_{k, i}$ of each $\Re_{k}^{(1)}$ via axon collaterals from the input source. The $i^{\text {th }}$ output from this avalanche is given by

$$
O_{i}(t)=\delta \sum_{k=1}^{K(\xi, T)} x_{k, i}(t)
$$

The following qualitative argument shows that the source vertex $v_{k, 1}$ in the $k^{\text {th }}$ outstar $\Re_{k}^{(1)}$ can be activated primarily in a time interval $[k \xi, k \xi+\lambda]$ of length less than $\xi$, and therefore $\mathfrak{T K}_{k}^{(1)}$ learns primarily the $k^{\text {th }}$ spatial approximation to the space-time pattern reaching $B_{n}$ at time $k \xi+\tau$.

First note that $\mathfrak{T l}_{k}^{(1)}$ cannot learn from the border inputs $I_{i}(t)$ in intervals of time $\left[t_{o}, T_{o}\right]$ for which $x_{1}(t-\tau) \cong 0$, since then by (35),

or

$$
\dot{z}_{k, 1 i}(t) \cong-u z_{k, 1 i}(t) \text {, }
$$

and thus by (34)

$$
z_{k, 1 i}(t) \cong e^{-u\left(t-t_{0}\right)} z_{k, 1 i}\left(t_{o}\right)
$$

$$
y_{k, 1 i}(t) \cong y_{k, 1 i}\left(t_{o}\right),
$$

whence no new learning has occurred. Supposing that $x_{o}$ and each $x_{k, 1}$ is initially zero, (32) implies that

$$
\begin{aligned}
x_{k, 1}(t) & =\beta_{0} e^{-\alpha_{1} t} \int_{0}^{t} e^{\alpha_{1} v} x_{0}(v-k \xi) d v \\
& = \begin{cases}0, & t \leqq k \xi, \\
\beta_{0} e^{-\alpha_{1}(t-k \xi)} \int_{0}^{t-k \xi} e^{\alpha_{1} v} x_{0}(v) d v, & t \geqq k \xi .\end{cases}
\end{aligned}
$$


Thus if $x_{o}(v)$ is large only in an interval of length $w<\xi$, then

$$
x_{k, 1}(t) \cong \beta_{0} e^{-\alpha_{1}(t-k \xi)} \Lambda
$$

for $t \geqq w+k \xi$, where

$$
\Lambda=\int_{0}^{w} e^{\alpha_{10} v} x_{0}(v) d v
$$

If moreover the decay rate $\alpha_{1}$ is large, (36) guarantees that

$$
x_{k, 1}(t) \cong 0
$$

for $t \geqq k \xi+\lambda$, where $w<\lambda<\xi$. If this can be achieved, then $\mathfrak{T}_{k}^{(1)}$ will learn only the inputs reaching $B_{n}$ within the interval $[k \xi+\tau, k \xi+\tau+\lambda]$; i.e., only the $k^{\text {th }}$ spatial approximation to the space-time pattern.

It remains only to check that $x_{o}(v)$ can be made large in an interval of length $w<\xi$. By (31),

$$
x_{0}(t)=e^{-\alpha_{0} t} \int_{0}^{t} e^{\alpha_{0} v} I_{0}(v) d v,
$$

so it suffices to make the duration of $I_{o}(v)$ less than $\lambda$ and to choose the decay rate $\alpha_{o}$ large.

This argument shows that a sequence of outstars can, at least crudely, learn a space-time pattern as a sequence of spatial patterns.

9. A better approximation: Signal thresholds eliminate source noise. The accuracy of learning by an outstar avalanche is limited by the fact that each $x_{k, 1}(t)>0$ for all $t \geqq t_{o}$ if $x_{k, 1}\left(t_{o}\right)>0, k=1,2, \cdots, K(\xi, T)$. Thus an outstar $\mathfrak{T K}_{k}^{(1)}$ can never wholly stop learning from the $(k+m)^{\text {th }}$ spatial approximations to the space-time pattern, $m=1,2, \cdots, K(\xi, T)-k$. The influence of the $(k+m)^{\text {th }}$ approximation will decrease as the source $x_{k, 1}(t)$ exponentially decays to ever smaller values, but some background interference can never be eliminated.

This difficulty can be readily overcome. We need merely guarantee that very small source values $x_{k, 1}(t)$ never create signals to border vertices. Thus we replace $\left({ }^{* *}\right)$ by the following system of equations.

$$
\begin{aligned}
\dot{x}_{0}(t) & =-\alpha_{0} x_{0}(t)+I_{0}(t) \\
\dot{x}_{k, 1}(t) & =-\alpha_{1} x_{k, 1}(t)+\beta_{0}\left[x_{0}(t-k \xi)-\Gamma_{0}\right]^{+} \\
\dot{x}_{i}(t) & =-\alpha x_{i}(t)+\beta \sum_{k=1}^{K(\xi, T)}\left[x_{k, 1}(t-\tau)-\Gamma_{1}\right]^{+} y_{k, l i}(t)+I_{i}(t) \\
y_{k, 1 i}(t) & =z_{k, 1 i}(t)\left[\sum_{m=2}^{n} z_{k, 1 m}(t)\right]^{-1}
\end{aligned}
$$


and

$$
\dot{z}_{k, 1 i}(t)=-u z_{k, 1 i}(t)+\gamma\left[x_{k, 1}(t-\tau)-\Gamma_{1}\right]^{+} x_{i}(t),
$$

where the signal thresholds $\Gamma_{o}$ and $\Gamma_{1}$ are positive, $k=1,2, \cdots, K(\xi, T)$, and $i=2, \cdots, n$. The system $(\ddagger)$ eliminates transmission of background noise from $v_{o}$ to each $v_{k, 1}$ and from $v_{k, 1}$ to each $v_{i}, i \neq 1$, by cutting off signals at the positive signal thresholds $\Gamma_{o}$ and $\Gamma_{1}$, respectively.

Alternatively the following system with $K(\xi, T)$ separate grids receiving axon collaterals from a common source can be used.

$$
\begin{aligned}
\dot{x}_{0}(t) & =-\alpha_{0} x_{0}(t)+I_{0}(t), \\
\dot{x}_{k, 1}(t) & =-\alpha_{1} x_{k, 1}(t)+\beta_{0}\left[x_{0}(t-k \xi)-\Gamma_{0}\right]^{+}, \\
\dot{x}_{k, i}(t) & =-\alpha x_{k, i}(t)+\beta\left[x_{k, 1}(t-\tau)-\Gamma_{1}\right]^{+} y_{k, 1 i}(t)+I_{i}(t),
\end{aligned}
$$

and

$$
y_{k, 1 i}(t)=z_{k, 1 i}(t)\left[\sum_{m=2}^{n} z_{k, 1 m}(t)\right]^{-1},
$$

where the $i^{\text {th }}$ output from this avalanche is

$$
O_{i}(t)=\delta \sum_{k=1}^{K(\xi, T)}\left[x_{k, i}(t)-\Gamma\right]^{+},
$$

with $\Gamma$ a small, but possibly positive, output threshold.

By (38) and (39), it is clear that an input of finite duration at $v_{o}$ will create a signal $\beta\left[x_{k, 1}(t-\tau)-\Gamma_{1}\right]^{+}$of finite duration in $e_{k, 1 i} \cdot(\ddagger)$ and $(\tilde{\ddagger})$ are constructed from $K(\xi, T)$ outstars with positive threshold $\Gamma_{1}$ (i.e., $\Gamma$-outstars), where below we write $\Gamma$ instead of $\Gamma_{1}$ for simplicity.

$$
\begin{aligned}
& \dot{x}_{1}(t)=-\alpha_{1} x_{1}(t)+I_{1}(t), \\
& \dot{x}_{i}(t)=-\alpha x_{i}(t)+\beta\left[x_{1}(t-\tau)-\Gamma\right]^{+} y_{1 i}(t)+I_{i}(t),
\end{aligned}
$$

$$
\begin{aligned}
& y_{1 i}(t)=z_{1 i}(t)\left[\sum_{m=2}^{n} z_{1 m}(t)\right]^{-1}, \\
& \dot{z}_{1 i}(t)=-u z_{1 i}(t)+\gamma\left[x_{1}(t-\tau)-\Gamma\right]^{+} x_{i}(t),
\end{aligned}
$$

$i=2, \cdots, n$. We must check that Theorem 1 holds in some form for ( $\ddagger)$. In fact the following theorem holds, in which we assume to avoid trivialities that the sums $x^{(1)}(t)=\sum_{m=2}^{n} x_{m}(t)$ and $z^{(1)}(t)=\sum_{m=2}^{n} z_{1 m}(t)$ are positive at $t=0$.

Theorem 2. Let $G^{(1)}, G^{(2)}, \cdots, G^{(N)}, \cdots$ be any sequence of $\Gamma$-outstars with 
equal but otherwise arbitrary initial data. Let $I_{i}^{(N)}$ be any inputs of the form

$$
I_{1}^{(N)}(t)=I_{1}(t) \chi\left(t-U_{1}(N)\right)+h_{N}(t)
$$

and

$$
I_{i}^{(N)}(t)=\theta_{i} I(t) \chi(t-U(N))
$$

$i=2, \cdots, n$, where

a) $\left\{\theta_{i}: i=2, \cdots, n\right\}$ is a fixed but arbitrary probability distribution;

b) $U_{1}(N)$ and $U(N)$ are any nonnegative and strictly increasing functions of $N \geqq 1$;

c) the inputs $I_{1}(t)$ and $I(t)$ are constrained only by the existence of positive constants $c$ and $T_{o}$ such that

$$
\int_{0}^{t} e^{-u(t-v)}\left[\int_{0}^{v} e^{-\alpha_{1}(v-\xi)} I_{1}(\xi) d \xi-\Gamma\right]^{+} d v \geqq c,
$$

and

$$
\int_{0}^{t} e^{-\alpha(t-v)} I(v) d v \geqq c
$$

for $t \geqq T_{o}$;

d) the input $h_{N}(t)$ is constrained only by being zero until $t>U(N)$.

\section{Then}

A) for every $N \geqq 1$, the limits $Q_{i}^{(N)}$ and $P_{1 i}^{(N)}$ exist,

B) for every $N \geqq 1$ and $t \geqq U(N), X_{i}^{(N)}(t)$ and $y_{1 i}^{(N)}(t)$ are monotonic and are contained in the interval $\left[m_{i}^{(N)}, M_{i}^{(N)}\right]$, where

$$
\lim _{N \rightarrow \infty} m_{i}^{(N)}=\lim _{N \rightarrow \infty} M_{i}^{(N)}=\theta_{i} .
$$

In particular, by (A) and (B),

$$
\lim _{N \rightarrow \infty} \lim _{t \rightarrow \infty} X_{i}^{(N)}(t)=\lim _{N \rightarrow \infty} \lim _{t \rightarrow \infty} y_{1 i}^{(N)}(t)=\theta_{i} ;
$$

C) for every $N \geqq 1$ and $i \neq 1$, the functions $\dot{y}_{1 i}^{(N)}, f_{i}^{(N)}$, and $g_{i}^{(N)}$ change sign at most once and not at all if $f_{i}^{(N)}(0) g_{i}^{(N)}(0) \geqq 0$. Moreover, $f_{i}^{(N)}(0) g_{i}^{(N)}(0)>0$ implies that $f_{i}^{(N)}(t) g_{i}^{(N)}(t)>0$ and that $y_{1 i}^{(N)}(t)$ is monotonic for all $t \geqq 0$.

Remark. If $\Gamma=0$, then condition (49) is weaker than condition (16). If $\Gamma>0$, then the limits $Q_{i}^{(N)}$ and $P_{i}^{(N)}$ are not generally equal.

Proof. The proof of Theorem 2 uses methods developed in [8] and [9]. The proof is divided into four sections. Section (I) transforms the nonlinear $\Gamma$-outstar into a linear system. Section (II) studies memory and recall in a $\Gamma$-outstar. Section (III) studies learning trials in a $\Gamma$-outstar that are not cut-off after a finite practice interval. Section (IV) cuts off learning after a finite practice interval and pastes the results of (II) onto the end of this interval. 
(I) A $\Gamma$-outstar becomes a linear system in terms of the probability distributions $y=\left\{y_{1 i}: i=2, \cdots, n\right\}$ and $X=\left\{X_{i}: i=2, \cdots, n\right\}$.

Lemma 1. The source function $x_{1}$ and the sums $x^{(1)}$ and $z^{(1)}$ depend on time only through the known inputs $I_{1}$ and $I$.

Proof. The assertion is obvious for $x_{1}$ by (43). Summing (44) and (45) over $i \neq 1$ yields

$$
\dot{x}^{(1)}=-\alpha x^{(1)}+\beta\left[x_{1}(t-\tau)-\Gamma\right]^{+}+I
$$

and

$$
\dot{z}^{(1)}=-u z^{(1)}+\gamma\left[x_{1}(t-\tau)-\Gamma\right]^{+} x^{(1)} .
$$

Integrating (43), (52), and (53) completes the proof.

Lemma 2. The probability distributions $y$ and $X$ satisfy the following linear system of equations.

$$
\dot{X}_{i}=A_{1}\left(y_{1 i}-X_{i}\right)+B_{1}\left(\theta_{i}-X_{i}\right)
$$

and

$$
\dot{y}_{1 i}=C_{1}\left(X_{i}-y_{1 i}\right)
$$

where

$$
\begin{aligned}
& A_{1}(t)=\frac{\beta\left[x_{1}(t-\tau)-\Gamma\right]^{+}}{x^{(1)}(t)}, \\
& B_{1}(t)=\frac{I(t)}{x^{(1)}(t)},
\end{aligned}
$$

and

$$
C_{1}(t)=\gamma\left[x_{1}(t-\tau)-\Gamma\right]^{+} \frac{x^{(1)}(t)}{z^{(1)}(t)},
$$

and are hence known functions of time by Lemma 1.

Proof. Since $X_{i}=x_{i} / x^{(1)}$,

$$
\dot{X}_{i}=\frac{1}{x^{(1)}}\left(\dot{x}_{i}-x_{i} \frac{\dot{x}^{(1)}}{x^{(1)}}\right)
$$

which yields (54) after substituting (44) and (52), cancelling and rearranging terms. Since $y_{1 i}=z_{1 i} / z^{(1)}$,

$$
\dot{y}_{1 i}=\frac{1}{z^{(1)}}\left(\dot{z}_{1 i}-z_{1 i} \frac{\dot{z}^{(1)}}{z^{(1)}}\right)
$$

which yields (55) after substituting (45) and (53), cancelling and rearranging terms. 
(II) Memory and recall experiments in $\left[t_{o}, \infty\right)$ are characterized by choosing $I_{i}(t)=0$ for $t \geqq t_{o}$ and $i=2, \cdots, n$.

Lemma 3. Let $(\ddagger)$ be given with arbitrary initial data in $\left[t_{o}-\tau, t_{o}\right]$, border inputs $I_{i}(t)=0$ for $t \geqq t_{0}$ and $i=2, \cdots, n$, and any input $I_{1}(t)$. If $x_{1}(t-\tau) \leqq \Gamma$ for $t$ in an interval $\left[t_{1}, t_{2}\right]$ such that $t_{o}<t_{1}<t_{2}$, then $y_{1 i}(t)$ and $X_{i}(t)$ are constant in $\left[t_{1}, t_{2}\right]$. If $x_{1}(t-\tau)>\Gamma$ for $t$ in $\left[t_{1}, t_{2}\right]$, then $y_{1 i}(t)$ and $X_{i}(t)$ are monotonic in opposite senses with $\left|y_{1 i}(t)-X_{i}(t)\right|$ monotone decreasing. Thus the limits

$$
Q_{i}=\lim _{t \rightarrow \infty} X_{i}(t)
$$

and

$$
P_{1 i}=\lim _{t \rightarrow \infty} y_{1 i}(t)
$$

exist, and $X_{i}(t)$ and $y_{1 i}(t)$ lie in the interval $\left[m_{i}, M_{i}\right]$ for $t \geqq t_{o}$, where $m_{i}=$ $\min \left(X_{i}\left(t_{o}\right), y_{1 i}\left(t_{o}\right)\right)$ and $M_{i}=\max \left(X_{i}\left(t_{o}\right), y_{1 i}\left(t_{o}\right)\right)$.

Proof. By hypothesis, $I(t)=0$ for $t \geqq t_{o}$. Hence (54) becomes

$$
\dot{X}_{i}=A_{1}\left(y_{1 i}-X_{i}\right) \text {. }
$$

The proof follows by inspection of (55) and (56) using the facts that $A_{1}$ and $C_{1}$ are nonnegative and continuous, and are positive or zero according as $x_{1}(t-$ $\tau)>\Gamma$ or $x_{1}(t-\tau) \leqq \Gamma$. (See [8], Theorem 1).

(III) Another change of variables in needed to study learning in ( $¥)$, namely from $y_{1 i}$ and $X_{i}$ to $f_{i}=y_{1 i}-X_{i}$ and $g_{i}=X_{i}-\theta_{i}$. Throughout this section, (49) and (50) will be assumed to hold.

Lemma 4. $f_{i}$ and $g_{i}$ satisfy the equations

$$
\dot{f}_{i}=-D_{1} f_{i}+B_{1} g_{i}
$$

and

$$
\dot{g}_{i}=-B_{1} g_{i}+A_{1} f_{i}
$$

where $D_{1}=A_{1}+C_{1}$.

Proof. Rewrite (54) and (55) in terms of $f_{i}$ and $g_{i}$.

The oscillations of $f_{i}$ and $g_{i}$ can be studied using the following lemma, in which $f=f_{i}, g=g_{i}, a=-D_{1}, b=B_{1}, c=A_{1}$, and $d=-B_{1}$.

Lemma 5. Let the functions $f$ and $g$ satisfy the differential equations

$$
\begin{aligned}
& \dot{f}=a f+b g \\
& \dot{g}=c f+d g,
\end{aligned}
$$

where $a, b, c$, and $d$ are continuous functions and the off-diagonal coefficients $b$ and c are nonnegative. Then $f$ and $g$ change sign at most once and not at all if $f(0) g(0) \geqq 0$. Moreover $f(0) g(0)>0$ implies $f(t) g(t)>0$ for all $t \geqq 0$. 
Lemma 5 is proved in [8], p. 663-664. By Lemma 5, two cases arise for large values of $t$. Either

A) $f_{i}(t) g_{i}(t)<0$ for all large $t$,

or

B) $f_{i}(t) g_{i}(t) \geqq 0$ for all large $t$.

If (A) holds, then by (57) and (58), $f_{i}$ and $g_{i}$ are monotonic in opposite senses for all large $t$. Thus the $\operatorname{limits}_{\lim _{t \rightarrow \infty}} f_{i}(t)$ and $\lim _{t \rightarrow \infty} g_{i}(t)$ exist, and hence the limits $Q_{i}=\lim _{t \rightarrow \infty} X_{i}(t)$ and $P_{1 i}=\lim _{t \rightarrow \infty} y_{1 i}(t)$ exist.

The existence of $P_{1 i}$ in both cases (A) and (B) follows by Lemma 5. (55) implies

$$
\dot{y}_{1 i}=C_{1} f_{i} \text {. }
$$

Since $C_{1} \geqq 0, \dot{y}_{1 i}$ changes sign at most once and not at all if $f_{i}(0) g_{i}(0) \geqq 0$. In particular, $y_{1 i}(t)$ is monotonic for large $t$, whence $P_{1 i}$ exists, $i=2, \cdots, n$.

Proving the existence of $Q_{i}=\lim _{t \rightarrow \infty} X_{i}(t)$ in Case (B) and the equation $P_{1 i}=Q_{i}=\theta_{i}$ in both cases requires the following estimates on $x^{(1)}$ and $z^{(1)}$

Lemma 6. If the inputs $I_{1}$ and I satisfy (49) and (50), then $x^{(1)}(t)$ and $z^{(1)}(t)$ are bounded from above and below by positive constants.

Proof. Integrating (52) yields

$$
x^{(1)}(t)=e^{-\alpha t}\left[x^{(1)}(0)+\int_{0}^{t} e^{\alpha v}\left(\beta\left[x_{1}(v-\tau)-\Gamma\right]^{+}+I(v)\right) d v\right],
$$

and thus

$$
x^{(1)}(t) \geqq \int_{0}^{t} e^{-\alpha(t-v)} I(v) d v,
$$

which by (50) yields

$$
x^{(1)}(t) \geqq c \text { for } t \geqq T_{\text {o }} .
$$

Since also $x^{(1)}(t)$ is positive and continuous in $\left[0, T_{o}\right], x^{(1)}(t)$ has a positive lower bound in $[0, \infty)$.

Integrating (53) yields

$$
z^{(1)}(t)=e^{-u t}\left[z^{(1)}(0)+\gamma \int_{0}^{t} e^{u v}\left[x_{1}(v-\tau)-\Gamma\right]^{+} x^{(1)}(v) d v\right],
$$

which by (59) implies

$$
\begin{aligned}
z^{(1)}(t) & \geqq \gamma c \int_{T_{0}}^{t} e^{-u(t-v)}\left[x_{1}(v-\tau)-\Gamma\right]^{+} d v \\
& =\gamma c e^{u \tau} \int_{T_{0}-\tau}^{t-\tau} e^{-u(t-v)}\left[x_{1}(v)-\Gamma\right]^{+} d v
\end{aligned}
$$


for $t \geqq T_{\circ}+\tau$. For any $w \geqq 0$ and $s \geqq w+T_{\circ}$, integrating (43) yields

$$
\begin{aligned}
& \int_{w}^{v} e^{-u(s-v)}\left[x_{1}(v)-\Gamma\right]^{+} d v \geqq \\
& \int_{w}^{v} e^{-u(\theta-v)}\left[\int_{0}^{v} e^{-\alpha_{1}(v-\xi)} I_{1}(\xi) d \xi-\Gamma\right]^{+} d v \geqq c-W e^{-u v}
\end{aligned}
$$

where

$$
W=\int_{0}^{w} e^{u_{v}}\left[\int_{0}^{v} e^{-\alpha_{1}(v-\xi)} I_{1}(\xi) d \xi-\Gamma\right]^{+} d v .
$$

Thus letting $w=T_{\circ}-\tau$ and $s=t-\tau$,

$$
z^{(1)}(t) \geqq \frac{\gamma c^{2} e^{u \tau}}{2} \text { for } t \geqq \tau+\frac{1}{u} \log \frac{2 W}{c} .
$$

Hence $z^{(1)}(t)$ has a positive lower bound in [0, $\left.\infty\right)$.

Upper bounds for $x^{(1)}$ and $z^{(1)}$ readily follow from the boundedness of $I_{1}$ and $I$.

These estimates can be used to show that $\ddot{y}_{1 i}(t)$ is bounded. (At points where a two-sided derivative does not exist because of the threshold cut-off of $x_{1}(t-\tau)$ by $\Gamma_{1}$ in (55), a one-sided derivative is intended.) By (54) and (55),

$$
\begin{aligned}
\left|\ddot{y}_{1 i}\right| & =\left|\dot{C}_{1}\left(X_{i}-y_{1 i}\right)+C_{1}\left(\dot{X}_{i}-\dot{y}_{1 i}\right)\right| \\
& \leqq 2\left|\dot{C}_{1}\right|+C_{1}\left(\left|\dot{X}_{i}\right|+\left|\dot{y}_{1 i}\right|\right) \\
& \leqq 2\left[\left|\dot{C}_{1}\right|+C_{1}\left(A_{1}+B_{1}+C_{1}\right)\right] .
\end{aligned}
$$

Lemma 6 and the boundedness of $I$ imply the boundedness of $A_{1}, B_{1}, C_{1}$, and $\dot{C}_{1}$, and hence the boundedness of $\ddot{y}_{1 i}$.

The boundedness of $\ddot{y}_{1 i}$ and the existence of $P_{1 i}$ imply that

$$
\lim _{t \rightarrow \infty} \dot{y}_{1 i}(t)=0
$$

([8], Lemma 4). Hence by (55),

$$
\lim _{t \rightarrow \infty} C_{1}(t)\left(X_{i}(t)-y_{1 i}(t)\right)=0 .
$$

In case (A), (61) implies that

$$
\left(Q_{i}-P_{1 i}\right) \lim _{t \rightarrow \infty} C_{1}(t)=0 .
$$

Thus either $Q_{i}=P_{1 i}$ or $\lim _{t \rightarrow \infty} C_{1}(t)=0$. If $\lim _{t \rightarrow \infty} C_{1}(t)=0$, then

$$
\lim _{t \rightarrow \infty}\left[x_{1}(t)-\Gamma\right]^{+}=0
$$

by Lemma 6 . But (62) implies

$$
\lim _{t \rightarrow \infty} \int_{0}^{t} e^{-u(t-v)}\left[x_{1}(v)-\Gamma\right]^{+} d v=0,
$$

which contradicts (49). Hence $Q_{i}=P_{1 i}$ in case (A). 
We now prove that $Q_{i}=P_{1 i}$ implies $Q_{i}=\theta_{i}$. Suppose not, and in particular let $Q_{i}>\theta_{i}$. Then there exists a $T$ such that $t \geqq T$ implies

$$
\theta_{i}-X_{i}(t) \leqq-\frac{1}{2}\left(Q_{i}-\theta_{i}\right) \text {. }
$$

We will also show that there exists a constant $K_{1}$ and a positive constant $K_{2}$ such that

$$
\int_{0}^{t} B_{1}(v) d v \geqq K_{1}+K_{2} t
$$

for all $t \geqq 0$. Thus for any $S \geqq T$ and $t \geqq S$,

$$
\begin{aligned}
\int_{S}^{t} B_{1}(v)\left(\theta_{i}-X_{i}(v)\right) d v & \leqq-\frac{1}{2}\left(Q_{i}-\theta_{i}\right) \int_{S}^{t} B_{1}(v) d v \\
& \leqq \frac{1}{2}\left(Q_{i}-\theta_{i}\right) \int_{0}^{S} B_{1}(v) d v-\frac{1}{2}\left(Q_{i}-\theta_{i}\right)\left(K_{1}+K_{2} t\right) .
\end{aligned}
$$

Now define $h_{i} \equiv A_{1}\left(y_{1 i}-X_{i}\right)$ and note that since $A_{1}$ is bounded and $P_{1 i}=$ $Q_{i}$, there exists an $S$ such that

$$
h_{i}(t) \leqq \frac{1}{4}\left(Q_{i}-\theta_{i}\right) K_{2}
$$

for $t \geqq S$. For such an $S$, integrating (54) from $S$ to any $t \geqq S$ yields

$$
X_{i}(t) \leqq G_{i}(S)-\frac{1}{4}\left(Q_{i}-\theta_{i}\right) K_{2} t
$$

where

$$
\begin{aligned}
G_{i}(S)= & X_{i}(S)+\frac{1}{2}\left(Q_{i}-\theta_{i}\right) \int_{0}^{S} B_{1}(v) d v \\
& -\frac{1}{2} K_{1}\left(Q_{i}-\theta_{i}\right)-\frac{1}{4}\left(Q_{i}-\theta_{i}\right) S,
\end{aligned}
$$

and thus by (64),

$$
-\infty=\lim _{t \rightarrow \infty} X_{i}(t) \geqq 0 .
$$

This contradiction shows $Q_{i} \leqq \theta_{i}$. The contradiction

$$
\infty=\lim _{t \rightarrow \infty} X_{i}(t) \leqq 1
$$

similarly follows if $Q_{i}<\theta_{i}$.

It remains only to prove (63). Denote the finite upper bound of $x^{(1)}(t)$ by $M$. Then by (50), for any $\epsilon>0$ and $t \geqq T_{o}$,

$$
x^{(1)}(t) \leqq e^{-\alpha t}\left[\epsilon+\frac{M}{c} \int_{0}^{t} e^{\alpha v} I(v) d v\right],
$$

or

$$
B_{1}(t) \geqq \frac{c}{M} \frac{d}{d t} \log \left[\epsilon+\frac{M}{c} \int_{0}^{t} e^{\alpha \nu} I(v) d v\right]
$$


which yields for $t \geqq T_{\text {o }}$,

$$
\int_{0}^{t} B_{1}(v) d v \geqq L \log \left[\epsilon+\frac{M}{c} \int_{0}^{t} e^{\alpha v} I(v) d v\right],
$$

where

$$
L^{-1}=\frac{M}{c} \log \left[\epsilon+\frac{M}{c} \int_{0}^{T_{0}} e^{\alpha v} I(v) d v\right]
$$

By (50),

$$
\begin{aligned}
\int_{0}^{t} B_{1}(v) d v & \geqq L \log \left(\epsilon+M e^{\alpha t}\right) \\
& \geqq L \log M+L \alpha t,
\end{aligned}
$$

which completes the proof by setting $K_{1}=L \log M$ and, $K_{2}=L \alpha$, and noting that $L$, and thus $K_{2}$, can be made positive by choosing $\epsilon$ sufficiently large.

It remains only to consider Case (B). Suppose for specificity that $f_{i}(t) \geqq 0$ and $g_{i}(t) \geqq 0$ for $t \geqq T_{1}$. (The case $f_{i}(t) \leqq 0$ and $g_{i}(t) \leqq 0$ can be similarly treated.) Then $y_{1 i}(t) \geqq X_{i}(t) \geqq \theta_{i}$ and $y_{1 i}(t)$ is monotone decreasing for $t \geqq T_{1}$. The equalities $\theta_{i}=Q_{i}=P_{1 i}$ therefore hold if $P_{1 i}=\theta_{i}$. It remains only to consider the case $P_{1 i}>\theta_{i}$.

Let $X_{i}^{(\theta)}=X_{i}-\theta_{i}$ and $y_{i}^{(\theta)}=y_{1 i}-\theta_{i}$. Then (54) and (55) become

$$
\dot{X}_{i}^{(\theta)}=A_{1}\left(y_{i}^{(\theta)}-X_{i}^{(\theta)}\right)-B_{1} X_{i}^{(\theta)}
$$

and

$$
\dot{y}_{i}^{(\theta)}=C_{1}\left(X_{i}^{(\theta)}-y_{i}^{(\theta)}\right),
$$

where $y_{i}^{(\theta)}(t) \geqq 0$ for $t \geqq T_{1}$.

Lemma 7. Suppose $y_{i}^{(\theta)}(t) \geqq X_{i}^{(\theta)}(t) \geqq 0$ for $t \geqq T_{1}$, where we can choose $T_{1} \geqq T_{\circ}$ without loss of generality. Then there exists $a \mu \varepsilon(0,1)$ and $a T_{2}=T_{2}(\mu)$ such that

$$
X_{i}^{(\theta)}(t) \leqq(1-\mu) y_{i}^{(\theta)}\left(t-T_{2}\right)
$$

Proof. Integrating (65) in $[T, t]$ yields

$$
X_{i}^{(\theta)}(t)=U_{i}^{(\theta)}(t, T)+V_{i}^{(\theta)}(t, T),
$$

where

$$
\begin{aligned}
& U_{i}^{(\theta)}(t, T)=X_{i}^{(\theta)}(T) Z^{-1}(t, T) \\
& V_{i}^{(\theta)}(t, T)=Z^{-1}(t, T) \int_{T}^{t} y_{i}^{(\theta)} A_{1} Z(v, T) d v,
\end{aligned}
$$

and

$$
Z(t, T)=\exp \left[\int_{T}^{t}\left(A_{1}+B_{1}\right) d w\right]
$$


Since $X_{i}^{(\theta)}(T) \leqq y_{i}^{(\theta)}(T)$ for $T \geqq T_{1},(69)$ implies

$$
U_{i}^{(\theta)}(t, T) \leqq y_{i}^{(\theta)}(T) Z^{-1}(t, T) .
$$

(71) is evaluated as follows.

$$
\begin{aligned}
A_{1}+B_{1} & =\frac{\beta\left[x_{1}(t-\tau)-\Gamma\right]^{+}+I}{x^{(1)}} \\
& =\frac{d}{d t} \log x^{(1)}+\alpha,
\end{aligned}
$$

and thus

$$
Z(t, T)=\frac{x^{(1)}(t) e^{\alpha t}}{x^{(1)}(T) e^{\alpha T}}
$$

(70) now becomes

$$
V_{i}^{(\theta)}(t, T)=\frac{1}{x^{(1)}(t) e^{\alpha t}} \int_{T}^{t} y_{i}^{(\theta)} A_{1} x^{(1)} e^{\alpha v} d v,
$$

and since $y_{i}^{(\theta)}(v) \leqq y_{i}^{(\theta)}(T)$ for $v \geqq T$,

$$
V^{(\theta)}(t, T) \leqq y_{i}^{(\theta)}(T) R(t, T)
$$

where

$$
R(t, T)=\frac{1}{x^{(1)}(t) e^{\alpha t}} \int_{T}^{t} A_{1} x^{(1)} e^{\alpha v} d v .
$$

Since $A_{1}=\beta\left[x_{1}(t-\tau)-\Gamma\right]^{+} / x^{(1)}$,

$$
R(t, T)=\frac{1}{x^{(1)}(t) e^{\alpha t}} \int_{T}^{t} \beta\left[x_{1}(v-\tau)-\Gamma\right]^{+} e^{\alpha v} d v,
$$

and since

$$
\begin{aligned}
\beta\left[x_{1}(v-\tau)-\Gamma\right]^{+} e^{\alpha \nu} & =e^{\alpha v}\left(\dot{x}^{(1)}+\alpha x^{(1)}-I\right) \\
& =\frac{d}{d v}\left(x^{(1)} e^{\alpha \nu}\right)-I e^{\alpha \nu}
\end{aligned}
$$

Combining (68), (72), (73), and (74) yields

$$
X_{i}^{(\theta)}(t) \leqq y_{i}^{(\theta)}(T) P(t, T),
$$

where

$$
P(t, T)=1-\frac{1}{x^{(1)}(t) e^{\alpha t}} \int_{T}^{t} I e^{\alpha v} d v \quad(>0)
$$


By Lemma 6, there exists a positive constant $M$ such that

$$
P(t, T) \leqq 1-M \int_{T}^{t} e^{-\alpha(t-v)} I(v) d v
$$

Since

$$
c \leqq\left(\int_{0}^{T}+\int_{T}^{t}\right) e^{-\alpha(t-v)} I(v) d v \leqq \frac{I}{\alpha}
$$

for $t \geqq T \geqq T_{o}$, where $I=\sup \{I(t): t \geqq 0\}(<\infty)$,

$$
\begin{aligned}
\int_{T}^{t} e^{-\alpha(t-v)} I(v) d v & \geqq c-\int_{0}^{T} e^{-\alpha(t-v)} I(v) d v \\
& \geqq c-\frac{I}{\alpha} e^{-\alpha(t-T)} .
\end{aligned}
$$

Thus there exists a $T_{2}$ such that

$$
\int_{T}^{t} e^{-\alpha(t-v)} I(v) d v \geqq \frac{c}{2}
$$

for $t \geqq T+T_{2}$, and

$$
P(t, T) \leqq 1-\mu, \quad \mu=\frac{1}{2} c M,
$$

which along with (75) completes the proof.

Lemma 7 will now be used to draw a contradiction if $P_{1 i}>\theta_{i}$ in Case (B).

Since $y_{1 i}$ decreases monotonically to $P_{1 i}$, there exists a $T_{3} \geqq T_{1}$ such that

$$
y_{1 i}\left(t-T_{2}\right)-y_{1 i}(t) \leqq \frac{\mu}{2}\left(P_{1 i}-\theta_{i}\right)
$$

for $t \geqq T_{4}+T_{5}$. Thus for $t \geqq T_{2}+T_{3}$,

$$
\begin{aligned}
y_{1 i}(t)-X_{i}(t) & =\left(y_{1 i}(t)-y_{1 i}\left(t-T_{2}\right)\right)+\left(y_{1 i}^{(\theta)}\left(t-T_{2}\right)-X_{i}^{(\theta)}(t)\right) \\
& \geqq-\frac{\mu}{2}\left(P_{1 i}-\theta_{i}\right)+y_{i}^{(\theta)}\left(t-T_{2}\right)-X_{i}^{(\theta)}(t),
\end{aligned}
$$

which by (67) yields

$$
\begin{aligned}
y_{1 i}(t)-X_{i}(t) & \geqq-\frac{\mu}{2}\left(P_{1 i}-\theta_{i}\right)+\mu\left(y_{1 i}\left(t-T_{2}\right)-\theta_{i}\right) \\
& \geqq \frac{\mu}{2}\left(P_{1 i}-\theta_{i}\right) \quad(>0) .
\end{aligned}
$$

Thus by $(61), \lim _{t \rightarrow \infty} C_{1}(t)=0$, which contradicts (49). Hence $P_{1 i}=Q_{i}=\theta_{i}$ in all cases.

(IV) To complete the proof, we must study the effects of cutting off the learning interval at a finite time that is followed by recall experiments (inputs to $v_{1}$ 
alone) and/or memory intervals free from inputs. Thus we consider $\Gamma$-outstars with inputs given by (47) and (48).

By (II), $y_{1 i}^{(N)}(t)$ and $X_{i}^{(N)}(t)$ are contained in $\left[m_{i}^{(N)}, M_{i}^{(N)}\right]$ for $t \geqq U(N)$. Two cases now arise.

Case 1. $U(N) \leqq U_{1}(N)$. By (III), $Q_{i}=P_{1 i}=\theta_{i}$. Since

$$
X_{i}^{(N)}(t)=X_{i}(t) \text { and } y_{1 i}^{(N)}(t)=y_{1 i}(t)
$$

for $t \varepsilon[0, U(N)]$,

$$
\lim _{N \rightarrow \infty} X_{i}^{(N)}(U(N))=\lim _{N \rightarrow \infty} y_{1 i}^{(N)}(U(N))=\theta_{i}
$$

and thus

$$
\lim _{N \rightarrow \infty} m_{i}^{(N)}=\lim _{N \rightarrow \infty} M_{i}^{(N)}=\theta_{i}
$$

In particular,

$$
\lim _{N \rightarrow \infty} \lim _{t \rightarrow \infty} X_{i}^{(N)}(t)=\lim _{N \rightarrow \infty} \lim _{t \rightarrow \infty} y_{1 i}^{(N)}(t)=\theta_{i} .
$$

Case 2. $U(N)>U_{1}(N)$. In this case we will find that $X_{i}^{(N)}(U(N))$ and $y_{1 i}^{(N)}(U(N))$ lie in $\left[m_{i \theta}^{(N)}, M_{i \theta}^{(N)}\right]$, where

$$
m_{i \theta}^{(N)}=\min \left\{\theta_{i}, X_{i}^{(N)}\left(U_{1}(N)\right), y_{1 i}^{(N)}\left(U_{1}(N)\right)\right\}
$$

and

$$
M_{i \theta}^{(N)}=\max \left\{\theta_{i}, X_{i}^{(N)}\left(U_{1}(N)\right), y_{1 i}^{(N)}\left(U_{1}(N)\right)\right\} .
$$

Then by (III),

$$
X_{i}^{(N)}\left(U_{1}(N)\right)=X_{i}\left(U_{1}(N)\right) \text { and } y_{1 i}^{(N)}\left(U_{1}(N)\right)=y_{1 i}\left(U_{1}(N)\right) \text {, }
$$

and since $Q_{i}=P_{1 i}=\theta_{i}$,

$$
\lim _{N \rightarrow \infty} X_{i}^{(N)}\left(U_{1}(N)\right)=\lim _{N \rightarrow \infty} y_{1 i}^{(N)}\left(U_{1}(N)\right)=\theta_{i},
$$

whence (77) and thus (78) follow.

For $t \varepsilon\left[U_{1}(N), U(N)\right]$,

$$
\dot{X}_{i}^{(N)}=A_{i}^{(N)}\left(y_{i}^{(N)}-X_{i}^{(N)}\right)+B_{i}^{(N)}\left(\theta_{i}-X_{i}^{(N)}\right)
$$

and

$$
\dot{y}_{1 i}^{(N)}=C_{1}^{(N)}\left(X_{i}^{(N)}-y_{i}^{(N)}\right) .
$$

Suppose $y_{1 i}^{(N)}\left(U_{1}(N)\right) \geqq X_{i}^{(N)}\left(U_{1}(N)\right) \geqq \theta_{i}$. Then $y_{1 i}^{(N)}(t) \geqq X_{i}^{(N)}(t) \geqq \theta_{i}$ and $y_{1 i}^{(N)}(t)$ is monotone nonincreasing for $t \geqq U_{1}(N)$. Suppose $y_{1 i}^{(N)}\left(U_{1}(N)\right) \geqq$ $X_{i}^{(N)}\left(U_{1}(N)\right)<\theta_{i}$. Then $y_{1 i}^{(N)}(t)$ is monotone nonincreasing and $X_{i}^{(N)}(t)$ is monotone nondecreasing until $X_{i}^{(N)}(t)=\theta_{i}$ at time $t=T$, after which $y_{1 i}^{(N)}(t) \geqq$ $X_{i}^{(N)}(t) \geqq \theta_{i}$ and $y_{1 i}^{(N)}(t)$ is monotone nonincreasing for $t \geqq T$. In both cases 
$X_{i}^{(N)}(U(N))$ and $y_{1 i}^{(N)}(U(N))$ lie in $\left[m_{i \theta}^{(N)}, M_{i \theta}^{(N)}\right]$. The two remaining cases can be similarly treated.

The oscillatory behavior of $\dot{y}_{1 i}^{(N)}, f_{i}^{(N)}$ and $g_{i}^{(N)}$ readily follows from Lemmas 3,4 , and 5 , and the above argument. Theorem 2 is hereby proved.

Theorem 2 holds in the special case when $I_{1}$ and $I$ are constructed from sequences of input pulses, where an input pulse is a nonnegative continuous function that is positive in a finite interval.

Corollary 1. Let

$$
I_{1}(t)=\sum_{k=1}^{\infty} J_{1}\left(t-t_{1}(k)\right)
$$

and

$$
I(t)=\sum_{k=1}^{\infty} J(t-t(k)),
$$

where $J_{1}$ and $J$ are input pulses that are positive in $\left(0, \lambda_{1}\right)$ and $(0, \lambda)$, respectively,

$$
\sup _{t} \int_{0}^{t} e^{-\alpha_{1}(t-v)} J_{1}(v) d v>\Gamma,
$$

and the sequences $\left\{t_{1}(k): k \geqq 1\right\}$ and $\{t(k): k \geqq 1\}$ satisfy

$$
\epsilon_{1} \leqq t_{1}(k+1)-t_{1}(k) \leqq \epsilon_{2}
$$

and

$$
\delta_{1} \leqq t(k+1)-t(k) \leqq \delta_{2}
$$

for some positive numbers $\epsilon_{1}, \epsilon_{2}, \delta_{1}$, and $\delta_{2}$. Theorem 2 holds for any such choice of $I_{1}$ and $I$.

The proof is obvious, amounting merely to showing that the exponentially weighted sum of an input pulse that is iterated with bounded spacing eventually has positive upper and lower bounds.

Corollary 1 shows that a single $\Gamma$-outstar will learn a spatial pattern perfectly if its source vertex $v_{1}$ receives inputs from a $v_{0}$ which is perturbed by a sequence of sufficiently intense input pulses with bounded spacing.

Corollary 2. Let

$$
I_{1}(t)=\left[\sum_{k=1}^{\infty} \int_{0}^{t} e^{-\alpha_{0}(t-v)} J_{0}\left(v-t_{0}(k)\right) d v-\Gamma_{0}\right]^{+}
$$

and

$$
I(t)=\sum_{k=1}^{\infty} J(t-t(k)),
$$

where $J_{\circ}$ and $J$ are input pulses that are positive in $\left(0, \lambda_{\circ}\right)$ and $(0, \lambda)$, respectively, 


$$
\sup \int_{0}^{t} e^{-\alpha_{1}(t-v)}\left[\int_{0}^{v} e^{-\alpha_{0}(v-\xi)} J_{0}(\xi) d \xi-\Gamma_{0}\right]^{+} d v>\Gamma,
$$

and the sequences $\left\{t_{o}(k): k \geqq 1\right\}$ and $\{t(k): k \geqq 1\}$ satisfy

$$
\epsilon_{1} \leqq t_{o}(k+1)-t_{o}(k) \leqq \epsilon_{2}
$$

and

$$
\delta_{1} \leqq t(k+1)-t(k) \leq \delta_{2}
$$

for some positive numbers $\epsilon_{1}, \epsilon_{2}, \delta_{1}$, and $\delta_{2}$. Theorem 2 holds for any such choice of $I_{1}$ and $I$.

Let $I_{1}, I_{2}, \cdots, I_{n}, \cdots$ be the nonoverlapping closed intervals of time in which $x_{1}(t-\tau) \leqq \Gamma_{1}$, and let $J_{1}, J_{2}, \cdots, J_{n}, \cdots$ be the complementary open intervals on which $x_{1}(t-\tau)>\Gamma_{1}$. (55) shows that no learning occurs in the $\Gamma$-outstar for $t \varepsilon \bigcup_{n=1}^{\infty} I_{n}$, since then $\dot{y}_{1 i}(t)=0$. Corollary 2 shows, nonetheless, that the $\Gamma$-outstar can learn any spatial pattern perfectly for $t \varepsilon \bigcup_{n=1}^{\infty} J_{n}$, if it is driven by a series of intense input pulses at $v_{o}$.

To learn a series of spatial patterns in $K(\xi, T)$ $\Gamma$-outstars, it remains only to guarantee that the source functions $x_{k, 1}(t-\tau)$ of successive outstars exceed $\Gamma_{1}$ in successive, nonoverlapping time intervals, and that these time intervals occur when the $k^{\text {th }}$ spatial approximation to the space-time pattern is arriving at $B_{n}$. The first condition is readily achieved by choosing $J_{o}$ so that $\xi>T$, where

$$
T=\sup \left\{t: \int_{0}^{t} e^{-\alpha_{1}(t-v)}\left[\int_{0}^{v} e^{-\alpha_{0}(v-\xi)} J_{0}(\xi) d \xi-\Gamma_{0}\right]^{+} d v>\Gamma_{1}\right\}
$$

The second condition requires that when the $(k+1)^{\text {th }}$ spatial approximation arrives at $B_{n}$, effects of the $k$ former spatial approximations and of inputs from $v_{m, 1}, m=1,2, \cdots, k$, shall have substantially decayed. This condition is implemented by increasing the decay rate $\alpha$ in $B_{n}$, or by decreasing

$$
\sup _{i} \max _{i}\left|\dot{\theta}_{i}(t)\right|
$$

in intervals of length $\xi$, or by decreasing $T / \xi$, etc. The decay rate of previous spatial approximants relative to the arrival speed of new spatial approximants is the rate-limiting factor determining accuracy of learning in the $\Gamma$-outstar avalanche. Another way of improving this accuracy is mentioned in Section 12, and uses an inhibitory feedback mechanism. Or independent grids can be used.

10. Simultaneous storage of patterns and multimodal learning. A network that can learn any number of space-time patterns on any number of finite grids can now readily be constructed from $\Gamma$-outstar avalanches. Just one control vertex $v_{m, o}$ is needed to activate the $m^{\text {th }}$ independent pattern. See Figure 6 .

The avalanche with control $v_{1, o}$ can learn from both grids ("modalities") $G_{1}$ and $G_{2}$. The time lags $\tau_{1}^{(1)}$ and $\tau_{1}^{(2)}$ for signals to travel from the source vertex 


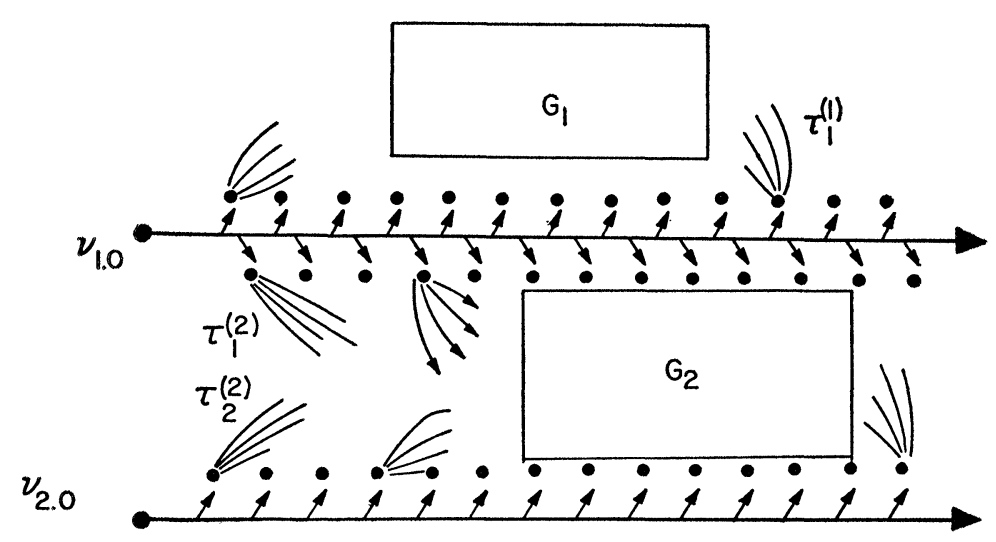

Figure 6.

$v_{1, k, 1}^{(1)}$ to $G_{1}$ and from $v_{1, k, 1}^{(2)}$ to $G_{2}$, respectively, can be different to help synchronize the learning of patterns in modalities which filter inputs at different rates. The avalanche with control $v_{2, o}$ also learns from $G_{2}$. Clearly the two avalanches will not interfere with each other if their controls $v_{1, o}$ and $v_{2, o}$ are perturbed at widely spaced times. For completeness, we list the equations of any number of avalanches learning from any number of grids.

$$
\begin{aligned}
\dot{x}_{m, o}(t)= & -\alpha_{m o} x_{m, o}(t)+I_{m, o}(t) \\
\dot{x}_{m k, 1}^{(r)}(t)= & -\alpha_{m 1}^{(r)} x_{m k, 1}^{(r)}(t)+\beta_{m o}^{(r)}\left[x_{m, o}\left(t-t_{m}^{(r)}-k \xi_{m}^{(r)}\right)-\Gamma_{m o}^{(r)}\right]^{+}, \\
\dot{x}_{i}^{(r)}(t)= & -\alpha^{(r)} x_{i}^{(r)}(t) \\
& +\sum_{m \varepsilon M_{r}} \sum_{k=1}^{k_{m r}} \beta_{m 1}^{(r)}\left[x_{m k, 1}^{(r)}\left(t-\tau_{m}^{(r)}\right)-\Gamma_{m 1}^{(r)}\right]^{+} y_{m k, 1 i}^{(r)}(t)+I_{i}^{(r)}(t), \\
y_{m k, 1 i}^{(r)}(t)= & z_{m k, 1 i}^{(r)}(t)\left[\sum_{p=2}^{n_{r}} z_{m k, 1 p}^{(r)}(t)\right]^{-1}
\end{aligned}
$$

and

$$
\dot{z}_{m k, 1 i}^{(r)}(t)=-u_{m}^{(r)} z_{m k, 1 i}^{(r)}(t)+\gamma_{m 1}^{(r)}\left[x_{m k, 1}^{(r)}\left(t-\tau_{m}^{(r)}\right)-\Gamma_{m 1}^{(r)}\right]^{+} x_{i}^{(r)}(t),
$$

where $x_{m, o}$ is the control vertex function of the $m^{\text {th }}$ avalanche, $x_{m k, 1}^{(r)}$ is the source vertex function of the $k^{\text {th }}$ spatial approximant in the $m^{\text {th }}$ avalanche lending to the $r^{\text {th }}$ grid, $x_{i}^{(r)}$ is the $i^{\text {th }}$ border vertex function of the $r^{\text {th }}$ grid, $y_{m k, 1 i}^{(r)}$ is the $(1, i)^{\text {th }}$ associational strength from the $m^{\text {th }}$ avalanche to the $r^{\text {th }}$ grid, etc. The avalanches indexed by $m \varepsilon M_{r}$ perturb the $r^{\text {th }}$ grid, which has $n_{r}$ vertices. The $m^{\text {th }}$ avalanche has $k_{m r}=K\left(\xi_{m}^{(r)}, T_{m}\right)$ spatial approximants leading to the $r^{\text {th }}$ grid. The relative onset time $t_{m}^{(r)}$ of the $m^{\text {th }}$ avalanche to the $r^{\text {th }}$ grid, and the time lags $\xi_{m}^{(r)}$ between successive spatial approximants can vary with $(r, m)$. If a given $\Gamma$-outstar sends signals only to a proper subset of a grid's vertices, then we partition the grid into two parts and consider each grid as a grid in its own right. 
An alternative to (79)-(83) is given by (79), (80), (82),

$$
\dot{x}_{m k, i}^{(r)}(t)=-\alpha_{m}^{(r)} x_{m k, i}^{(r)}(t)+\beta_{m 1}^{(r)}\left[x_{m k, 1}^{(r)}\left(t-\tau_{m}^{(r)}\right)-\Gamma_{m 1}^{(r)}\right]^{+} y_{m k, 1 i}^{(r)}(t)+I_{i}^{(r)}(t),
$$

and

$$
\dot{z}_{m k, 1 i}^{(r)}(t)=-u_{m}^{(r)} z_{m k, 1 i}^{(r)}(t)+\gamma_{m 1}^{(r)}\left[x_{m k, 1}^{(r)}\left(t-\tau_{m}^{(r)}\right)-\Gamma_{m 1}^{(r)}\right]^{+} x_{m k, i}^{(r)}(t) .
$$

In this case, each $\Gamma$-outstar $\left(\mathscr{T}^{(1)}\right)_{m k}^{(r)}$ in the $m^{\text {th }}$ avalanche has its own grid, which is perturbed by the inputs $I_{i}^{(r)}, i=2, \cdots, n_{r}$, via axon collaterals from the $r^{\text {th }}$ input source. The output to the $r^{\text {th }}$ output sink is given by

$$
O_{i}^{(r)}(t)=\delta^{(r)} \sum_{m \varepsilon M_{r}} \sum_{k=1}^{k_{m r}}\left[x_{m k, i}^{(r)}(t)-\Gamma_{m}^{(r)}\right]^{+}
$$

where the $\Gamma_{m}^{(r)}$ are small, but possibly positive, signal thresholds.

11. Diffuse arousal inputs. Several presentations, or trials, of a space-time pattern are often required before the pattern can be well learned by a $\Gamma$-outstar avalanche. Suppose that on the $i^{\text {th }}$ trial, the first spatial approximation to the pattern arrives $S_{i}$ time units after the control vertex $v_{o}$ emits a signal. Unless all $S_{i}$ are approximately equal, a given outstar $\mathfrak{T}_{k}^{(1)}$ in the avalanche will learn different spatial approximations on different trials, and the avalanche will never learn any one space-time pattern well. A way must be found, therefore, to guarantee that $v_{o}$ transmits a signal only if the relative timing of $v_{o}$ signal and $B_{n}$ input is approximately the same on all trials. This problem does not, of course, arise when $B_{n}$ receives a single spatial pattern throughout the time interval of length $T$ during which the signal from $v_{o}$ is active.

Our goal can be reached in either of two ways. The first way supposes that two sources of inputs to $v_{0}$ exist: a "conditioned stimulus", or CS, to $v_{0}$ alone, such as we have previously discussed, and a "diffuse arousal input", or DAI, which is controlled by the input sources that create the pattern at $B_{n}$. We can easily guarantee that

a) $x_{o}$ will exceed $\Gamma_{o}$, and therefore transmit a signal, only if both the CS and the DAI arrive at $v_{0}$ almost simultaneously, and

b) the DAI arrives at $v_{o}$ a prescribed time $\eta$ before the "unconditioned stimulus" arrives at $B_{n}$. Thus the term "arousal" in DAI means that a control vertex perturbed by a CS and a DAI readies its avalanche to be able to learn from the inputs reaching the grid a short time later. $\eta$ is chosen so that a signal from $v_{0}$ can activate the "synaptic knobs" of the first spatial approximant in the avalanche before the space-time pattern reaches the grid.

Given (a) and (b), we must also require

c) the input to $v_{0}$ on a recall trial is larger than the $C S$ to $v_{0}$ on a learning trial, or else no output from $B_{n}$ could ever occur during recall.

The DAI must also have the following properties. 
d) Every edge producing an input to $B_{n}$ must send a DAI, either directly or indirectly, to every control vertex $v_{m, o}$. Otherwise there would exist patterns playing on a small number of grid vertices which could never produce a DAI, and control vertices $v_{m, o}$ which could never send signals. Thus the term "diffuse" in DAI refers to a widespread spatial dispersion of this input to control vertices.

e) If the parameters of two avalanches differ, then the parameters of the DAI must differ commensurately. For example, let two avalanches with control vertices $v_{1, o}$ and $v_{2, o}$ be given such that $\Gamma_{1 o} \gg \Gamma_{2 o}$. If the same DAI perturbs both avalanches, then by choosing a DAI such that $\Gamma_{10}>x_{1,0}=x_{2,0}>\Gamma_{20}$, (a) will be satisfied in the first avalanche and not in the second. The DAI perturbing $v_{2,0}$ must therefore be chosen smaller than the DAI perturbing $v_{1,0}$.

Consider a single avalanche with control $v_{o}$ and border vertices $v_{i}$. The simplest choice of the DAI to $v_{o}$ created by the input source perturbing $v_{i}$ is

$$
\tilde{I}_{i o}(t)=\omega I_{i}(t+\eta),
$$

and the total DAI is

$$
\begin{aligned}
\tilde{I}_{0}(t) & =\sum_{i=2}^{n} \tilde{I}_{i 0}(t) \\
& =\omega \sum_{i=2}^{n} I_{i}(t+\eta),
\end{aligned}
$$

where $\omega$ and $\eta$ are positive. In other words, $v_{o}$ receives a suitable multiple of the total input received by the border $\eta$ time units later. $\eta$ is chosen to satisfy (b) and $\omega$ is chosen to satisfy (a). By (a), neither a CS $I_{o}$ nor a DAI $\tilde{I}_{o}$ alone can exceed $\Gamma_{o}$, but a suitable intense combination $I_{0}+\tilde{I}_{0}$ can. That is,

$$
\tilde{I}_{0} \equiv \sup _{t} \int_{0}^{t} e^{-\alpha_{0}(t-v)} I_{0}(v) d v<\Gamma_{0}
$$

and

$$
\omega \sup _{t} \sum_{i=2}^{n} \int_{0}^{t} e^{-\alpha_{0}(t-v)} I_{i}(v+\eta) d v<\Gamma_{0}
$$

whereas

$$
\sup _{t} \int_{0}^{t} e^{-\alpha_{0}(t-v)}\left[I_{0}(v)+\omega \sum_{i=2}^{n} I_{i}(v+\eta)\right] d v>\Gamma_{0}
$$

for sufficiently intense inputs. Other things equal, $\omega$ must decrease as $n$ increases to satisfy (86). Suppose that $n$ is large. Since an input to just one border vertex $v_{i}$ is a spatial pattern, an intense input to such a vertex, summating as $\omega I_{i}(t+\eta)$ with the CS $I_{o}(t)$ at $v_{o}$, ought to satisfy (87). Since $\omega$ is very small for large $n$, $\omega I_{i}(t+\eta)$ will be small as well, and thus $\hat{I}_{o}$ in (85) will lie close to $\Gamma_{o}$ for large $n$. The individual DAI's $\omega I_{i}(t+\eta)$ are therefore small "subliminal" contributions to the total input at $v_{0}$ which suffice to drive $x_{0}$ to suprathreshold values. See Figure 7. 

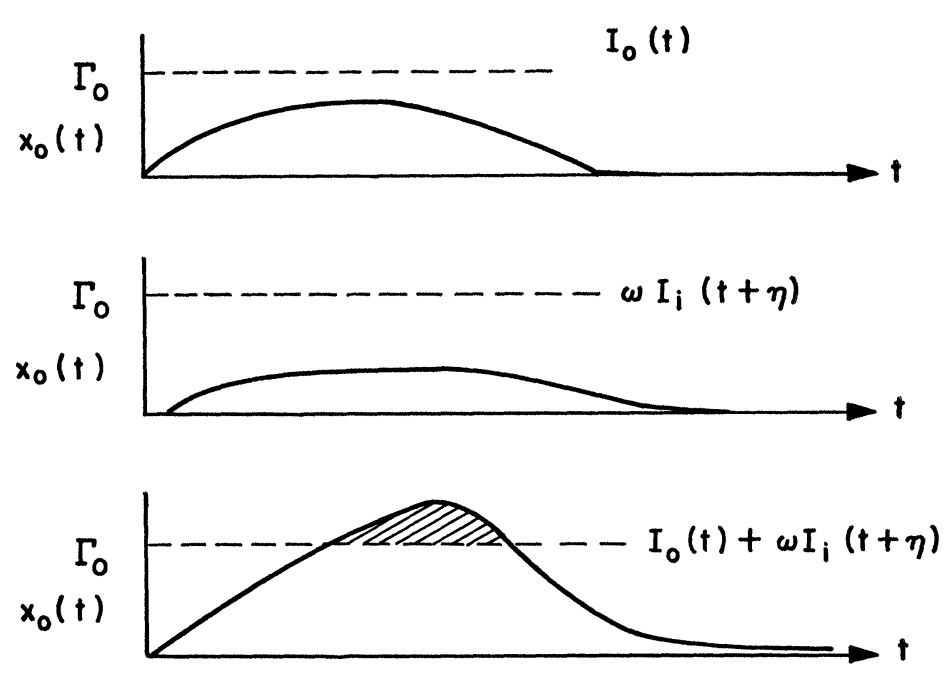

Frgure 7.

A second way to accomplish the same goals is to let $v_{0}$ be inhibited by a "diffuse suppression input", or DSI, which is, in turn, inhibited by the input source that perturbs $B_{n} \eta$ time units before $B_{n}$ is perturbed. Many statements about summating excitatory influences to exceed threshold can be replaced by statements concerning inhibition of inhibiting influences ("disinhibition") that had prevented thresholds from being reached.

12. Feedback inhibition from control vertices. Once $v_{0}$ emits a brief signal due to summation of a CS and a DAI, no new signals can be allowed to leave $v_{0}$ until all $K(\xi, T)$ outstars in the avalanche have been excited by the signal. Otherwise, the second signal from $v_{o}$ would again cause each $\Gamma$-outstar in the avalanche to learn from more than one spatial approximation to the pattern. Thus the DAI sent towards $v_{o}$ by later spatial approximations to the pattern at $B_{n}$ must never reach $v_{o}$. The signal from $v_{o}$ itself must therefore inhibit these later inputs. A release from inhibition will automatically occur after the signal from $v_{0}$ has excited the entire avalanche. See Figure 8.

Figure 8 interpolates a vertex $v_{+1}$ between the DAI input and $v_{o}$, which is inhibited by a feedback input from $v_{-1}$. The "+" signs designate "excitatory" edges and the "-" signs designate "inhibitory" edges. This particular version of feedback inhibition of the DAI by $v_{o}$ satisfies equations of the form

$$
\begin{aligned}
\dot{x}_{0}(t) & =-\alpha_{0} x_{0}(t)+I_{0}(t)+\beta_{+1}\left[x_{+1}\left(t-\tau_{+1}\right)-\Gamma_{+1}\right]^{+}, \\
\dot{x}_{-1}(t) & =-\alpha_{-1} x_{-1}(t)+\beta_{0}\left[x_{0}\left(t-\xi_{0}\right)-\Gamma_{0}\right]^{+},
\end{aligned}
$$

and

$$
\dot{x}_{+1}(t)=-\alpha_{+1} x_{+1}(t)-\beta_{-1}\left[x_{-1}\left(t-\xi_{-1}\right)-\Gamma_{-1}\right]^{+}+\omega \sum_{i=2}^{n} I_{i}(t+\eta),
$$




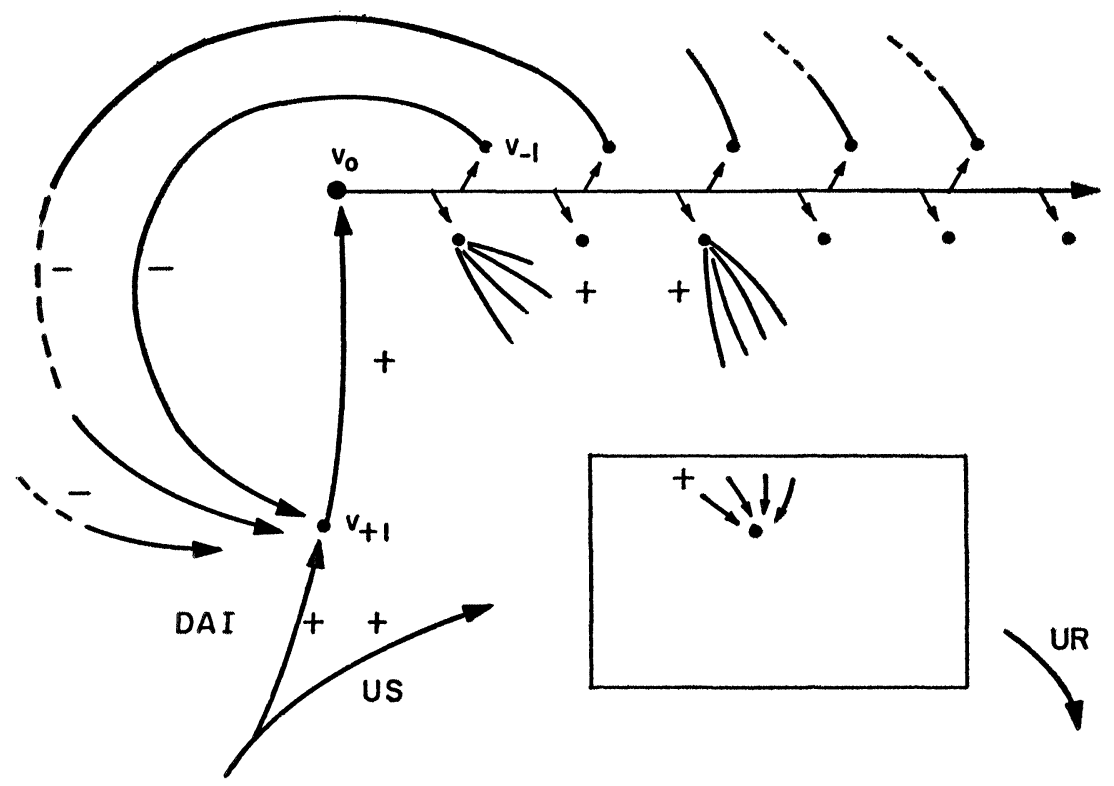

Figure 8.

where $\omega$ and $\eta$ must be adjusted to compensate for the additional parameters and time lags in (88)-(90). The reader can readily construct improvisations on this theme, say without the inhibitory internode $v_{-1}$, and can modify the conditions (85)-(87) accordingly.

A very interesting phenomenon occurs if Figure 8 is replaced by Figure 9 .

Then the DAI input is inhibited periodically and, for suitable parameter choices, never reaches a size needed to create a second signal from $v_{0}$ until the spacetime pattern has been wholly delivered. But also the pattern input to $B_{n}$ is periodically inhibited, and the period of successive inhibitory signals can be chosen equal to $\xi$. These inhibitory signals chop up the incoming space-time pattern into spatial approximations to the pattern with a time spacing of $\xi$ units between successive approximations, and thereby prepare the pattern for learning by successive $\Gamma$-outstars in the avalanche. This chopping procedure enables the avalanche to learn spatial approximations to rapidly varying $\theta_{i}(t)$ given relatively small values of $\alpha$, since it decreases the length of time intervals during which large inputs reach $B_{n}$. During a recall trial, the periodic inhibitory signals do not affect the grid. Thus the small decay parameter $\alpha$ allows the successively activated spatial approximants to blend smoothly on the grid, and to thereby produce a more smoothly modulated output through time than is encapsulated in the remembered successive spatial approximations of the avalanche.

13. A hierarchy of facilitatory and incompatible behavioral acts. Suppose that two avalanches perturb the same grid and have each learned different 


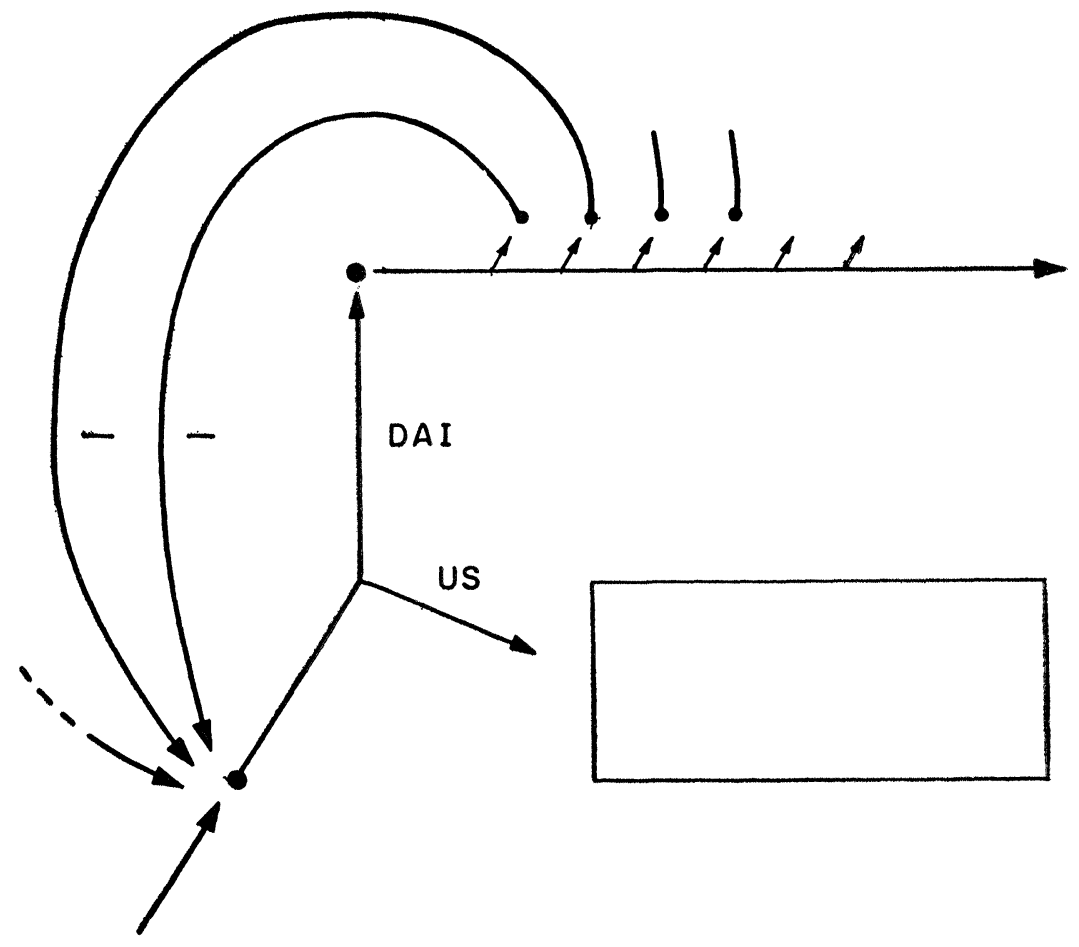

Frgure 9.

space-time patterns. These avalanches must be prevented from thereafter perturbing the common grid simultaneously. Otherwise, the grid output will be a noisy mixture of both patterns, and the memory of each avalanche will be destroyed. This can easily be guaranteed by supposing either that the control vertices $v_{1, o}$ and $v_{2, o}$ of the two avalanches mutually inhibit each other, or that the CS to one control vertex sends an inhibitory axon collateral to the other. See Figure 10.
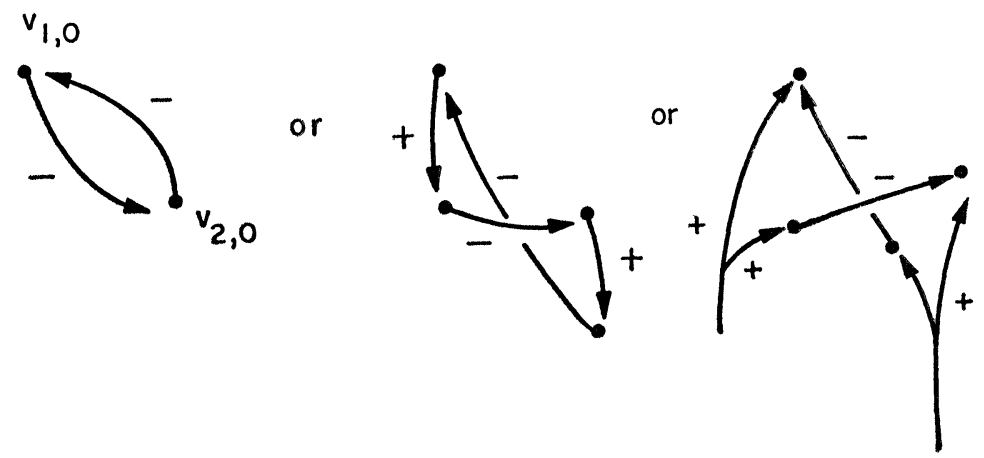

Figure 10. 
A similar competition between control vertices occurs in a signed outstar ([3], Section 15).

The mechanisms of Figure 9 and 10 taken together have the following effect. Suppose that no inputs activate $v_{1, o}$ or $v_{2, o}$ for some time, so that $x_{1, o}$ and $x_{2, o}$ are approximately zero. Then let $v_{1, o}$ receive a large simultaneous CS and DAI (and much larger than the CS + DAI received by $v_{2, o}$ ). By Figure 10, $v_{1, o}$ inhibits $v_{2, o}$ and simultaneously sends a signal to its avalanche. By Figure 9, this signal can be made to cut off the DAI's reaching $v_{2, o}$ until the first avalanche has been played out. This mechanism guarantees that at most one avalanche will learn from, or perform upon, the grid at any time. The avalanches $v_{1, o}$ and $v_{2, o}$ are said to control "incompatible" behavioral acts, because the performance of one interferes with the performance of the other. Usage of the same grid can be thought of in a behavioral context as control of the same muscle groups, or way-stations to the same muscle groups.

Avalanches that control different grids need not mutually inhibit each other, since performances of space-time patterns on different grids (i.e., by different "muscles") need not interfere with one another (one can talk as one walks). The spatial distribution of inhibitory connections between control vertices determines the degree of "incompatibility" between the behavioral acts controlled by the vertices. If for example, $v_{1, o}$ inhibits $v_{2, o}$ much more strongly than $v_{2, o}$ inhibits $v_{1, o}$, then $v_{1, o}$ is the dominant control of the pair, since it will activate its avalanche and inhibit $v_{2, o}$ if both receive equal inputs. In any given network, a hierarchy of incompatible controls playing on common grids will be determined by the relative strengths of mutual inhibition between the control vertices.

In a similar fashion, controls operating over different grids can facilitate one another by contributing to each other's DAI. For example, suppose that $v_{1,0}$ and $v_{2, o}$ perform upon different grids, and $v_{1, o}$ sends a small excitatory signal to $v_{2, o}$ whenever $x_{1, o}$ reaches suprathreshold values. Then $v_{2, o}$ will be able to activate its avalanche if it simultaneously receives a US. For example let $v_{1,0}$ control "sniffing" in response to a DAI caused by "hunger" and the US caused by the smell of "food", and let $v_{2, o}$ control "salivation". The above remarks suggest that $\mathfrak{T}$ will start "salivating" after "sniffing" begins with less provocation than before "sniffing" begins. In this sense, prior search for food has "lowered Ir's salivation threshold." Actually "sniffing" has raised $x_{2,0}$ closer to its signal threshold. This example is admittedly quite naive, but it can be extended to achieve rather realistic effects.

In short, the geometry of the signed graph with fixed excitatory and inhibitory path weights between IT's control vertices determines hierarchies of incompatible, facilitatory, and merely unrelated output behaviors by $\mathfrak{T}$. The avalanches within $\mathfrak{N}$ that succeed in learning or performing at any time will be determined by the spatial distribution of all conditioned, urconditioned, and diffuse arousal stimuli to $\Re$, along with the feedback excitatory and inhibitory signals thereby created. The geometry of the signed graph can automatically forbid the simultaneous occurrence of incompatible behaviors and can favor the 
performance of those behaviors which are most compatible with T's internal states at any time.

14. Novelty and habituation. A "novel stimulus" in this setting has a clear meaning. It is an input that succeeds in activating some avalanche. Essentially all other inputs are too weak, or will be suppressed by feedback inhibition, at some stage in their development. All inputs to a grid give rise to a DAI, but the DAI will be effective in producing learning only if some avalanche is "ready" to take heed of it, because of prior preparation by a CS - that is, by other inputs that determine the network's "psychological set" at the given time. Even if a spatial pattern is novel at a given time, repeating it will cause "habituation"that is, once an avalanche is activated, it will suppress the DAI of the repeated pattern.

15. Higher-order controls. The above remarks can be extended in many directions. For example, a given set of control vertices $v_{k, o}$ can be the border vertices of a $\Gamma$-outstar $\operatorname{Tr}_{0}^{(1)}$ whose input to $v_{k, o}$ is the DAI of $v_{k, o}$. See Figure 11.

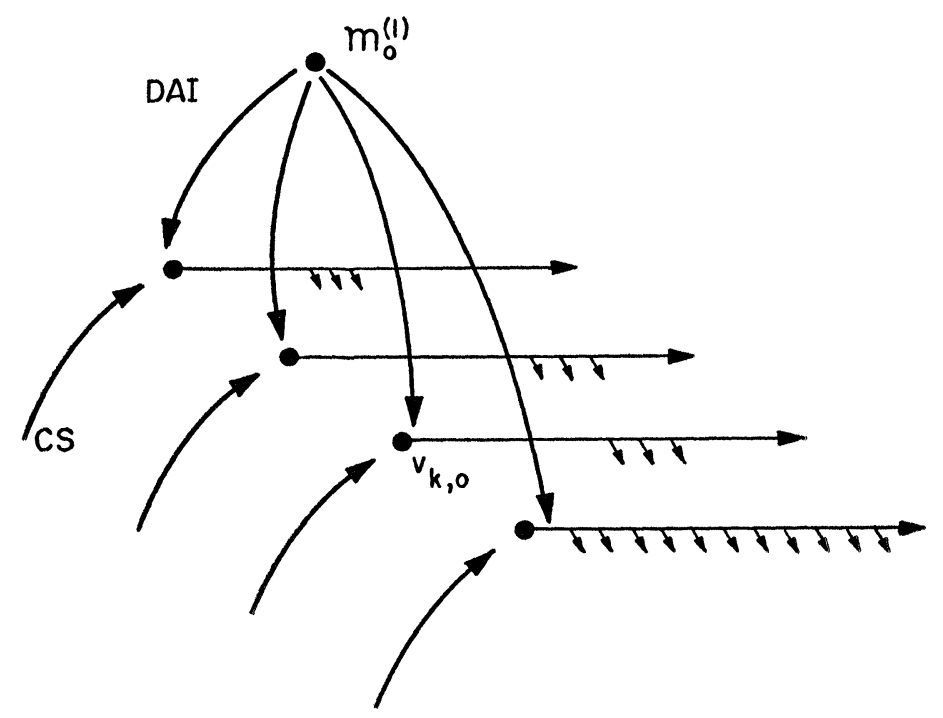

Figure 11.

After several trials, the associational strengths $y_{o, 1 k}$ of $\mathfrak{T}_{0}^{(1)}$ can learn any spatial pattern of CS's playing on the control vertices $v_{k, o}$. A later recall input to just the control vertex of $\mathfrak{T K}_{0}^{(1)}$ can therefore activate a spatial pattern of spacetime patterns.

16. "On" - "Off" performance of complicated reflexes and "paying attention". The equations for a $\Gamma$-outstar avalanche can be modified so that no 
learning occurs, and an arbitrarily prescribed "reflex pattern" is produced whenever the control is activated. Merely replace (83) by the equation

$$
\dot{z}_{m k, i}^{(r)}(t)=0 ;
$$

that is, choose the parameters $u_{j k}=v_{i k}=0$ in (3). Then the system (79)-(82), and (91) can reproduce any number of reflexes, parameterized by $m$, on any number of grids parameterized by $r$. The $m^{\text {th }}$ reflex to the $r^{\text {th }}$ grid will be determined by the sequence $\left\{y_{m}^{(r)}(k)\right\}$ of spatial approximants with

$$
y_{m}^{(r)}(k)=\left(y_{m k, 12}^{(r)}(0), y_{m k, 13}^{(r)}(0), \cdots, y_{m k, i n_{r}}^{(r)}\right),
$$

$k=1,2, \cdots, K\left(\xi_{m}^{(r)}, T_{m}\right)$, where $T_{m}$ is the duration of the $m^{\text {th }}$ reflex. Speaking heuristically, activating the single "control neuron" $v_{m, o}$ can reproduce a very complicated prescribed sequence of inputs to any collection of muscle groups, and can thereby produce very complicated motor behavior. It is, in fact, well known that a single neuron can activate complicated reflex acts, say in insects ([17], p. 8).

If the reflex is repetitive, as in walking, then we let the control $v_{m, o}$ excite itself via an axon collateral, thereby forming a loop, as in Figure 12.

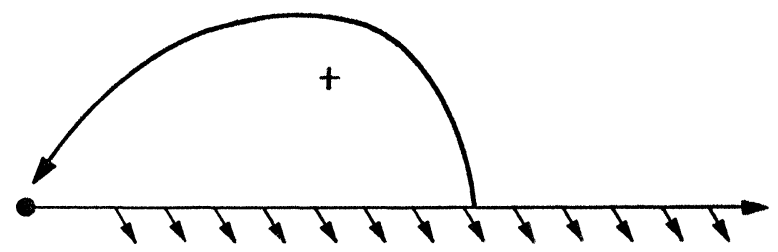

Figure 12.

Each total activation of the loop corresponds to a single cycle of the reflex. One can easily choose the parameters of the loop collateral such that its input alone will not activate another cycle of the avalanche. In this case, supplementary inputs must bring $x_{m, o}$ to suprathreshold values-for example, DAI's due to IT's motion through the environment. If the parameters are chosen so that the loop collateral can activate $x_{m, o}$ alone, then "walking" will continue until $\mathfrak{T}$ sends an inhibitory signal to $v_{m, o}$ and thereby breaks the cycle. In this case, "walking" can be viewed as a simple "on"-"off" phenomenon: once the control $v_{m, o}$ is turned on, the cyclic performance of the motor pattern described by (92) can be carried out automatically until $\mathfrak{T}$ shuts the reflex off with an inhibitory input. During the interim period, IT need never worry about the "walking" process, albeit the pattern (92) can be a very complicated one. TC can "pay attention" to other matters entirely.

A simpler case is that of a "clasp" or "clutch" reflex; for example, a hand closed tightly around an object for a long time. Here, each successive spatial approximant $y_{m}^{(r)}(k)$, in (92) can be chosen the same-i.e., is an iterated spatial pattern activating the clutching muscle groups. This clutch pattern can be continued 
indefinitely by chosing the feedback loop in Figure 12 so that the time lag needed for the feedback loop to re-excite $v_{0}$ is approximately equal to the time lag needed to finish up one cycle of the clutch reflex. Thus the second cycle can begin just as the cycle ends, unless an inhibitory signal $v_{0}$ drives $x_{0}$ to subthreshold values. Sets of outstar avalanches can, in principle, perform much more complicated patterns, such as learning any number of pieces of piano music. Of course, they will perform the pieces without any artistic subtlety.

17. Alternative systems. Part II of this paper [13] will study learning of space-time patterns by avalanches constructed from the following $\Gamma$-outstar.

$$
\begin{aligned}
& \dot{x}_{1}(t)=-\alpha x_{1}(t)+I_{1}(t), \\
& \dot{x}_{i}(t)=-\alpha x_{i}(t)+\beta\left[x_{1}(t-\tau)-\Gamma_{1}\right]^{+} z_{1 i}(t)+I_{i}(t),
\end{aligned}
$$

and

$$
\dot{z}_{1 i}(t)=-u z_{1 i}(t)+\gamma\left[x_{1}(t-\tau)-\Gamma_{1}\right]^{+} x_{i}(t),
$$

$i=2, \cdots, n$. This $\Gamma$-outstar does not use the ratios $y_{1 i}(t)$. It is therefore more plausible physically, but also its memory decays exponentially at the rate $u$. The ratios

$$
y_{1 i}(t)=z_{1 i}(t)\left[\sum_{m=2}^{n} z_{1 m}(t)\right]^{-1}
$$

nonetheless are still remembered essentially perfectly in the absence of practice.

Part II will also discuss avalanches constructed from a $\Gamma$-outstar satisfying (92), (93) and

$$
\dot{z}_{1 i}(t)=\left[-u z_{1 i}(t)+\gamma x_{i}(t)\right]\left[x_{1}(t-\tau)-\Gamma_{1}\right]^{+} .
$$

This system has perfect memory except during recall trials, and is also plausible physically. During recall trials, this system undergoes "extinction" of memory. (95) replaces the decay rate $u$ in (94) by

$$
u\left[x_{1}(t-\tau)-\Gamma_{1}\right]^{+} .
$$

(96) couples the decay of transmitter producing activity to the presynaptic spiking frequency. Were this coupling to exist in vivo, it would presumably be carried out by the increase in $\mathrm{Na}^{+}$and the decrease in $\mathrm{K}^{+}$within $\mathrm{N}_{1 i}$ that occurs during the action potential.

Actually Part II will discuss a large class of closely related equations that include (92)-(94) and (92), (95), (96) as special cases.

\section{REFERENCES}

[1] S. Grossberg, Some physiological and biochemical consequences of psychological postulates, Proc. Natl. Acad. Sci., 60 (1968) 758-765.

[2] S. Grossberg, Embedding fields: A theory of learning with physiological implications, J. Math. Psych., 6 (1969). 
[3] S. Grossberg, On learning, information, lateral inhibition, and transmitters, Math. Biosci., 4 (1969).

[4] S. Grossberg, On the production and release of chemical transmitters and related topics in cellular control, J. Theoret. Biol., 22 (1969) 325-364.

[5] S. Grossberg, On the serial learning of lists, Math. Biosci., 4 (1969).

[6] S. Grossberg, Nonlinear difference-differential equations in prediction and learning theory, Proc. Natl. Acad. Sci., 58 (1967) 1329-1334.

[7] S. Grossberg, Some nonlinear networks capable of learning a spatial pattern of arbitrary complexity, Proc. Natl. Acad. Sci., 59 (1968) 368-372.

[8] S. Grossberg, A prediction theory for some nonlinear functional-differential equations, I-Learning of lists, J. Math. Anal. and Applics., 21 (1968) 643-694.

[9] S. Grossbera, A prediction theory for some nonlinear functional-differential equations, II-Learning of Patterns, J. Math. Anal. and Applics., 22 (1968) 490-522.

[10] S. Grossberg, On the global limits and oscillations of a system of nonlinear differential equations describing a flow on a probabilistic network, J. Diff. Eqns., 5 (1969) 531-563.

[11] S. Grossberg, On the variational systems of some nonlinear difference-differential equations, J. Diff. Eqns., in press.

[12] S. Grossberg, On learning of spatiotemporal patterns by networks with ordered sensory and motor components, I-Excitatory components of the cerebellum, Studies in Applied Math, 48 (1969).

[13] S. Grossberg, Some networks that can learn, remember, and reproduce any number of complicated space-time patterns, II, submitted to SIAM J. Appl. Math.

[14] R. B. Livingston, Brain mechanisms in conditioning and learning, Neurosciences Research Symposium Summaries, Vol. 2 (ed., Sснмгтт, F. O., et al.), M. I. T. Press, Cambridge, 1967.

[15] S. R. KonEy (ed.), The Biology of Myelin, Hoeber-Harper, New York, 1959.

[16] E. C. Crosby, T. Humphrey, \& E. W. LAUER, Correlative Anatomy of the Nervous System, The Macmillan Company, New York, 1962.

[17] V. G. Dethier, Physiology of Insect Senses, Methuen and Company, London, 1963.

Massachusetts Institute of Technology Date communicated: November 26, 1968 